ARTICLE

DOI: $10.1038 / s 41467-018-04851-z$

\title{
ATR is required to complete meiotic recombination in mice
}

\author{
Sarai Pacheco ${ }^{1,2}$, Andros Maldonado-Linares ${ }^{1,2}$, Marina Marcet-Ortega ${ }^{1,2}$, Cristina Rojas ${ }^{1,2}$, \\ Ana Martínez-Marchal 1,2, Judit Fuentes-Lazaro, ${ }^{1,2}$, Julian Lange ${ }^{3,4}$, Maria Jasin ${ }^{5}$, Scott Keeney (i) 3,4, \\ Oscar Fernández-Capetillo ${ }^{6}$, Montserrat Garcia-Caldés ${ }^{1,2}$ \& Ignasi Roig (10 1,2
}

Precise execution of recombination during meiosis is essential for forming chromosomallybalanced gametes. Meiotic recombination initiates with the formation and resection of DNA double-strand breaks (DSBs). Cellular responses to meiotic DSBs are critical for efficient repair and quality control, but molecular features of these remain poorly understood, particularly in mammals. Here we report that the DNA damage response protein kinase ATR is crucial for meiotic recombination and completion of meiotic prophase in mice. Using a hypomorphic Atr mutation and pharmacological inhibition of ATR in vivo and in cultured spermatocytes, we show that ATR, through its effector kinase CHK1, promotes efficient RAD51 and DMC1 assembly at RPA-coated resected DSB sites and establishment of interhomolog connections during meiosis. Furthermore, our findings suggest that ATR promotes local accumulation of recombination markers on unsynapsed axes during meiotic prophase to favor homologous chromosome synapsis. These data reveal that ATR plays multiple roles in mammalian meiotic recombination.

\footnotetext{
${ }^{1}$ Genome Integrity and Instability Group, Institut de Biotecnologia i Biomedicina, Universitat Autònoma de Barcelona, Cerdanyola del Vallès 08193, Spain.

${ }^{2}$ Department of Cell Biology, Physiology and Immunology, Universitat Autònoma de Barcelona, Cerdanyola del Vallès 08193, Spain. ${ }^{3}$ Molecular Biology Program, Memorial Sloan Kettering Cancer Center, New York, NY 10065, USA. ${ }^{4}$ Howard Hughes Medical Institute, Memorial Sloan Kettering Cancer Center, New York, NY 10065, USA. ${ }^{5}$ Developmental Biology Program, Memorial Sloan Kettering Cancer Center, New York, NY 10065, USA. ${ }^{6}$ Genomic Instability Group, Spanish National Cancer Research Centre, Madrid 28029, Spain. Correspondence and requests for materials should be addressed to I.R. (email: ignasi.roig@uab.cat)
} 
B efore the first meiotic division, hundreds of programmed DNA double-strand breaks (DSBs) are formed throughout the genome by the SPO11 protein ${ }^{1}$. The repair of these lesions by homologous recombination promotes homologous chromosome synapsis and crossover formation. Crossovers are essential for proper chromosome segregation and thus for preventing aneuploidy in gametes ${ }^{2}$.

The cellular machinery that senses meiotic DSBs and regulates their repair is similar to the surveillance proteins that monitor DNA integrity in somatic cells and that, as a result of DNA damage, activate repair pathways, arrest cell cycle progression, and, if necessary, induce apoptosis ${ }^{3,4}$. A major one of these regulators in mice is the ataxia telangiectasia mutated and Rad-3 related (ATR) protein kinase ${ }^{3,4}$. ATR is activated by the presence of single-stranded DNA (ssDNA), which mainly arises from stalled replication forks or resected DSBs. ssDNA is rapidly covered by the ssDNA-binding protein complex RPA, which recruits ATR and its cofactors. ATR activation promotes stabilization and restart of stalled replications forks, DNA repair, and cell cycle arrest ${ }^{3}$.

In mammalian meiosis, ATR accumulates on unsynapsed chromosome axes, such as the heterologous parts of the $\mathrm{X}$ and $\mathrm{Y}$ chromosomes 5 . This accumulation causes chromatin alterations that, in males, condense the sex chromosomes into a distinct chromatin domain known as the sex body. Such heterochromatinization leads to the transcriptional silencing of these regions, referred to as meiotic sex chromosome inactivation (MSCI) ${ }^{6}$. MSCI is indispensable for the completion of meiotic prophase, as sex-body failure provokes a mid-prophase arrest ${ }^{7}$. Although DSBs may contribute to this mode of ATR action, DSB formation is not strictly required as ATR can promote a similar response to unsynapsed axes in the absence of meiotic DSBs ${ }^{8,9}$.

Whether ATR also functions in response to meiotic DSBs has remained obscure. In yeast, the ATR ortholog Mec1 is involved in directing meiotic cell cycle progression, promoting meiotic recombination, and controlling crossover formation ${ }^{10}$. In plants, ATR also promotes meiotic recombination by regulating recombinase DMC1 deposition at resected $\mathrm{DSBs}^{11}$. In mouse spermatocytes, ATR and its cofactors co-localize with other DNA repair proteins at sites undergoing homologous recombination, suggesting that ATR functions in meiotic recombination ${ }^{12}$. However, this hypothesis has not been formally tested, in part because abolition of Atr expression is embryonically lethal ${ }^{13}$. We overcame this challenge in this study by diminishing ATR function using genetic and pharmacological tools.

Our findings revealed previously invisible functions for ATR in proper loading of strand-exchange proteins at DSBs, correct timing of crossover formation, elongation of the synaptonemal complex (SC, the zipper-like proteinaceous structure that juxtaposes homologous chromosomes), and proper accumulation of recombination markers on the axes of unsynapsed chromosomes.

\section{Results}

Using the Seckel mouse model to study meiotic ATR functions. We used a previously described hypomorphic mutation that reduces ATR expression in most tissues ${ }^{14}$. This allele mimics a mutation found in some human patients with Seckel syndrome (OMIM 210600), which is characterized by dwarfism, microcephaly, mental retardation, and a beak-like nose ${ }^{15}$. The mutation is a synonymous $A$ to $G$ transition in exon 9 that causes frequent skipping of this exon during splicing, thereby severely reducing Atr expression ${ }^{14,16}$. In adult male mice homozygous for this "Seckel" allele $\left(A t r^{S}\right)$, whole-testis Atr mRNA - but not protein levels were substantially reduced, as previously reported ${ }^{14}$ (Fig. 1a and Supplementary Fig. 1a). Importantly, we were unable to detect by immunoblot the $\sim 70 \mathrm{kDa}$-truncated ATR protein expected from the exon 9-deleted transcript in Seckel mouse testis (Supplementary Fig. 1a). Moreover, even if expressed, the truncated ATR form would be unlikely to compensate for ATR function because it would lack the kinase catalytic domain. Testes from adult $A t r^{S / S}$ mice were much smaller than wild type (26.0 \pm $11.1 \%$ of wild type, at $2-4$ months of age, mean $\pm \mathrm{SD}, N=20$ ). This difference may be attributable to the dwarfism of Seckel mice $^{14}$ rather than a meiotic arrest. Indeed, the cellular composition of seminiferous tubules appeared grossly normal (Supplementary Fig. 1b) and a previous study found that $A t^{S / S}$ mice produce functional sperm ${ }^{14}$.

Meiotic prophase stages of surface-spread spermatocytes can be determined by the cytological patterns of axis and SC components. At leptonema, SYCP3 protein begins to form the axis of each chromosome (Supplementary Fig. 1c). Synapsis begins at zygonema and leads to the formation of the SC containing SYCP1. At pachynema, autosomal homologous chromosomes are fully synapsed and SYCP3 and SYCP1 proteins completely co-localize. At diplonema, SCs disassemble but homologous chromosomes remain held together by chiasmata. Cytological evaluation of $A t r / S$ germ cells revealed that both the total percent of cells in meiotic prophase and the fractions of meiotic cells within each prophase stage were similar to wild type (Supplementary Fig. 1d). Taken together, these results show that Atr ${ }^{S / S}$ cells are capable of progressing through meiosis and completing spermatogenesis.

We sought evidence of reduced ATR function in $A t r S / S$ spermatocytes. The only known meiotic role of ATR is in sexbody formation and MSCI at pachynema ${ }^{5,7}$. Since sex-body formation was indistinguishable in wild type and Seckel mice (wild type: $0.4 \pm 0.6 \%, N=318 ; A t r^{S / S}: 2.3 \pm 1.2 \%, N=389 ; p=$ 0.183 , one-way ANOVA), we analyzed the localization pattern of ATR and the intensity of four ATR-dependent sex-body markers in pachytene $A t r / S$ spermatocytes (Fig. 1b, c and Supplementary Fig. 1e). ATR localizes to the unsynapsed axes of the sex chromosomes, where it phosphorylates the histone $\mathrm{H} 2 \mathrm{AX}$ to form $\gamma \mathrm{H} 2 \mathrm{AX}$, and HORMAD2 on S271 (pHORMAD2), among other proteins ${ }^{5}$. With the help of MDC1, ATR spreads to the XY chromatin, where it continues to phosphorylate $\mathrm{H} 2 \mathrm{AX}^{17}$. Finally, other sex-body markers such as SUMO-1 are incorporated into the XY chromatin ${ }^{17}$. In wild type, most cells contained ATR localized to both the chromatin and the axes of the sex chromosomes. In contrast, most $A t r^{S / S}$ cells displayed ATR only on the XY axes. The staining pattern in Seckel cells for the ATRdependent sex-body markers studied $(\gamma \mathrm{H} 2 \mathrm{AX}$, pHORMAD2, MDC1, and SUMO-1, see examples for $\gamma \mathrm{H} 2 \mathrm{AX}$ in Fig. 1d, e) was indistinguishable from control cells. However, we observed a significant change in total signal intensity in $A t r S / S$ compared with wild type for each of them. Notably, although most $A t r S / S$ cells lacked ATR in XY chromatin, we detected the other sexbody markers there. Signal intensities of $\gamma \mathrm{H} 2 \mathrm{AX}$ and SUMO-1 were reduced, but PHORMAD2 and MDC1 signal were higher, possibly reflecting a compensatory mechanism. Despite these alterations, MSCI appears to be unaffected, as the X-linked gene $Z f x$ was efficiently silenced in pachytene $A t r S / S$ spermatocytes (Supplementary Fig. 1f, g). We conclude that wild-type levels of ATR function are not needed to initiate and/or maintain MSCI. Importantly, however, our findings demonstrate that homozygosity for the Seckel mutation causes mild changes in ATR function in spermatocytes.

To further test this conclusion, we analysed other known ATR target proteins in the sex body. ATR phosphorylates multiple proteins involved in DNA repair, cell cycle control or replication origin firing ${ }^{3}$. Some of these are phosphorylated in the sex body, although it is not clear if they have any MSCI function, like 
a

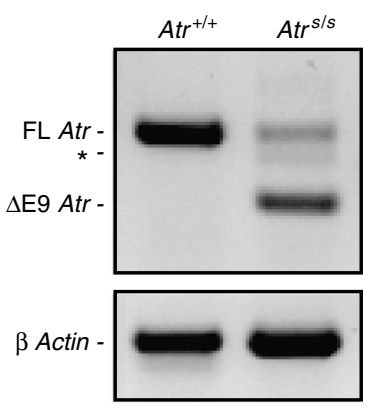

b

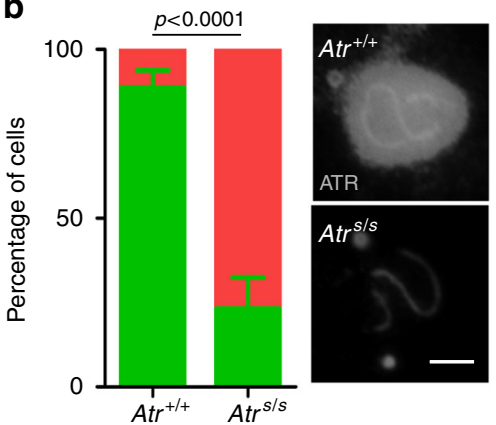

C

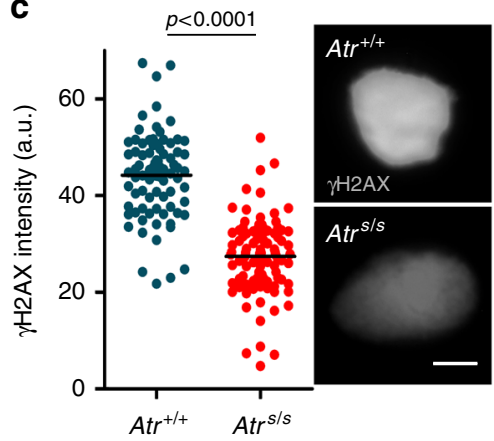

ATR signal on all $\mathrm{XY}$ chromatin ATR signal confined to $X Y$ axes

d Leptonema Zygonema

Early pachynema Late pachynema Diplonema
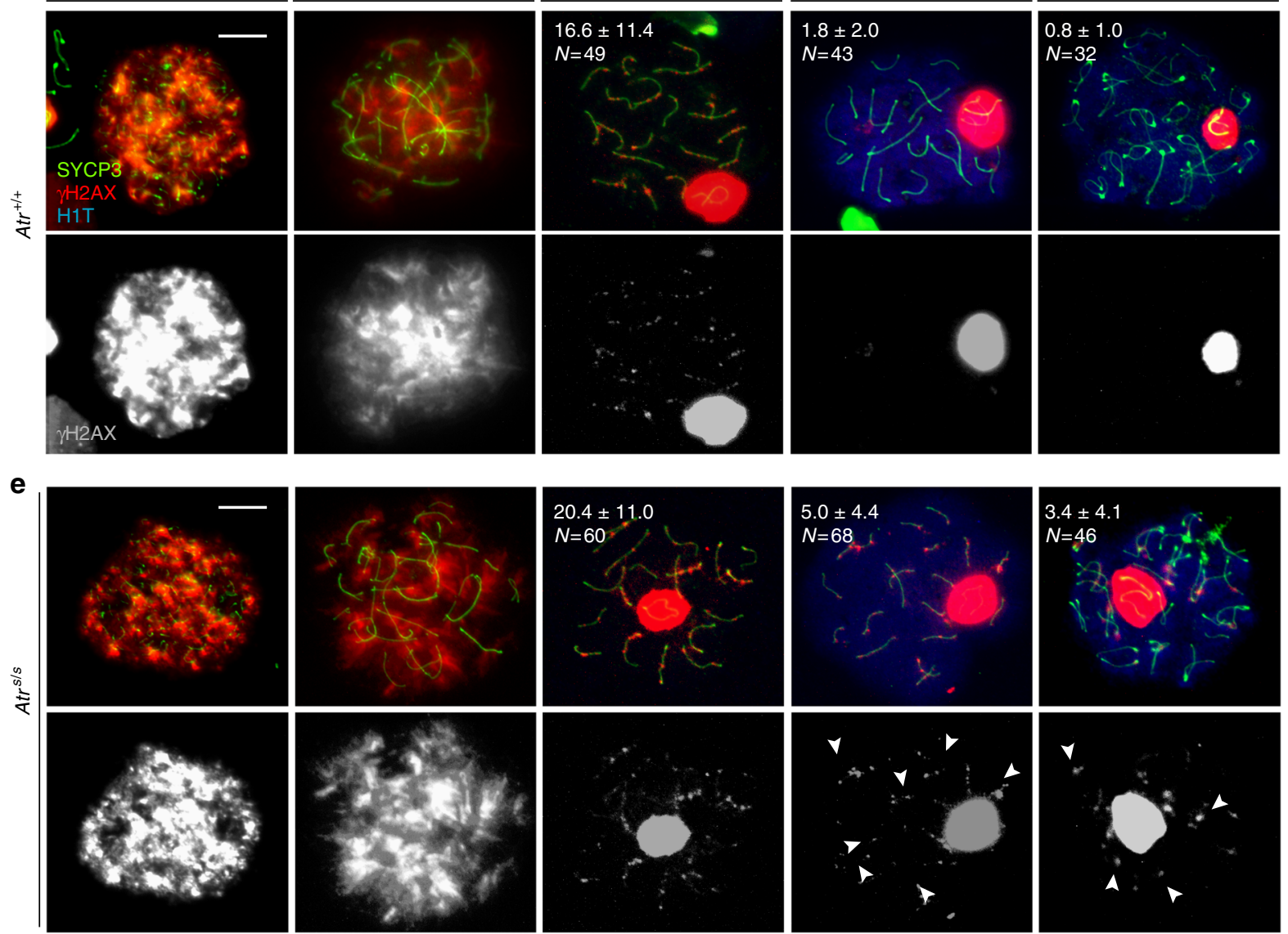

Fig. 1 Seckel mouse spermatocytes exhibit more $\gamma \mathrm{H} 2 \mathrm{AX}$ patches than wild-type cells. a RT-PCR using primers that anneal to Atr exons 8 and 10 . Atr $\mathrm{S} / \mathrm{S}$ testis exhibits two main RT-PCR products, one corresponding to full-length Atr (FL Atr, $477 \mathrm{bp}$ ), which is substantially reduced, and another corresponding to Atr lacking exon 9 ( $\triangle \mathrm{E} 9$ Atr, $284 \mathrm{bp}$ ). Asterisk denotes RT-PCR product resulting from the use of a cryptic splice donor site ${ }^{16}$. RT-PCR for $\beta$ Actin is also provided as a control. $\mathbf{b}$ Percentage of cells exhibiting ATR staining extended to $\mathrm{X}$ and $\mathrm{Y}$ chromatin or confined to the $\mathrm{X}$ and $\mathrm{Y}$ chromosome axes in $A$ tr $+/+$ and $\operatorname{Atr}^{S / S}(N=284$ and $N=282$, respectively). Columns and lines indicate the mean and standard deviation (SD) from analysis performed on three wildtype and three mutant mice. Images show ATR straining on the sex body in $\mathrm{Atr}^{+/+}$and $\mathrm{Atr}^{\mathrm{S} / \mathrm{S}}$ pachytene spermatocytes. Images were captured with the same exposure time. Scale bar $=2 \mu \mathrm{m}$. c Quantification of the intensity of $\gamma \mathrm{H} 2 \mathrm{AX}$ staining on the sex body in arbitrary units (a.u.). Horizontal black lines denote the means. Images show representative sex bodies from $\mathrm{Atr}^{+/+}$and $\mathrm{Atr} / \mathrm{S}$ pachytene spermatocytes immunostained against $\gamma \mathrm{H} 2 \mathrm{AX}$. Images were captured with the same exposure time. Scale bar $=2 \mu \mathrm{m}$. Representative images of $A t r+/+(\mathbf{d})$ and $A t r S / S$ (e) spermatocytes at different stages of meiotic prophase immunostained against SYCP3, $\gamma \mathrm{H} 2 \mathrm{AX}$, and $\mathrm{H} 1 \mathrm{~T}$. Scale bars $=10 \mu \mathrm{m}$. Numbers represent the mean $\pm \mathrm{SD}$ of $\gamma \mathrm{H} 2 \mathrm{AX}$ patches found in each stage and genotype. $N$ denotes the number of cells analyzed. H1T incorporation marks spermatocytes after mid-pachynema ${ }^{62}$. Arrowheads indicate examples of aberrant autosomal $\gamma \mathrm{H} 2 \mathrm{AX}$ patches in $A t r^{S / S}$ spermatocytes. The differences between controls and Atr ${ }^{S / S}$ for $\gamma \mathrm{H} 2 \mathrm{AX}$ patch numbers at late pachynema and diplonema were statistically significant $(p=0.0001$ and $p=0.0007$, respectively, $t$ test $)$ 
phosphorylation of RPA32 on S33 (pRPA) or phosphorylation of CHK1 on S317 (pCHK1) ${ }^{18}$. Both markers were altered in Seckel mouse cells. Whereas pRPA in wild-type cells was almost exclusively found evenly covering the chromatin of the $\mathrm{X}$ and $\mathrm{Y}$ chromosomes, $\sim 30 \%$ of $A t r^{S / S}$ spermatocytes showed pRPA staining that accumulated more predominantly in the vicinity of the $\mathrm{X}$ and $\mathrm{Y}$ axes, without covering the whole $\mathrm{XY}$ chromatin (Supplementary Fig. 1h). Similarly, about half of $\mathrm{Atr}^{\mathrm{S} / \mathrm{S}}$ spermatocytes had a similar, more axis-restricted staining pattern for pCHK1, unlike the broader chromatin staining seen in wild type (Supplementary Fig. 1h). Moreover, overall pCHK1 intensity was visibly higher in wild-type cells. These phosphorylated forms thus resemble the localization of ATR itself in Seckel mouse cells (Fig. 1b). These findings corroborate that the Seckel mutation impairs ATR function in spermatocytes.

a
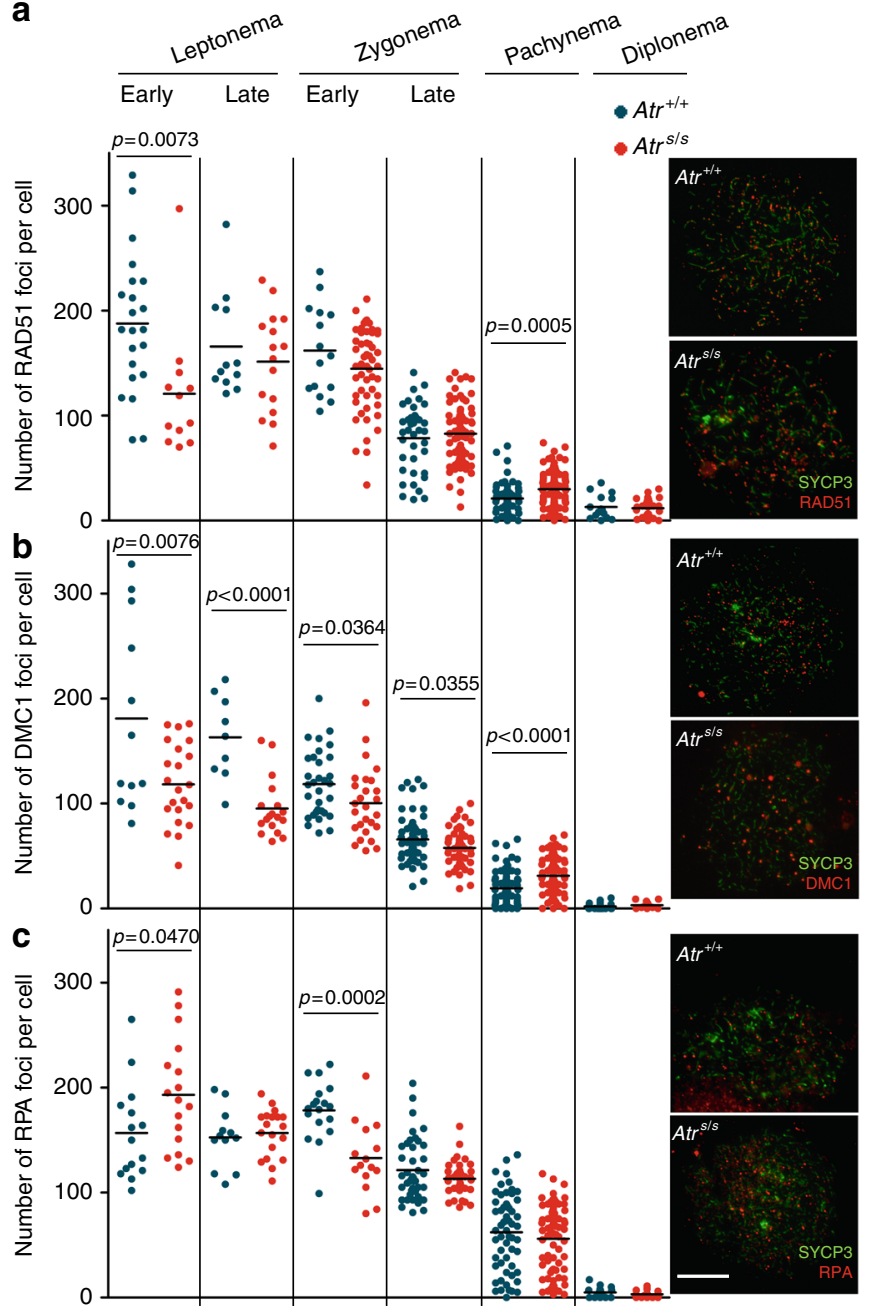

d
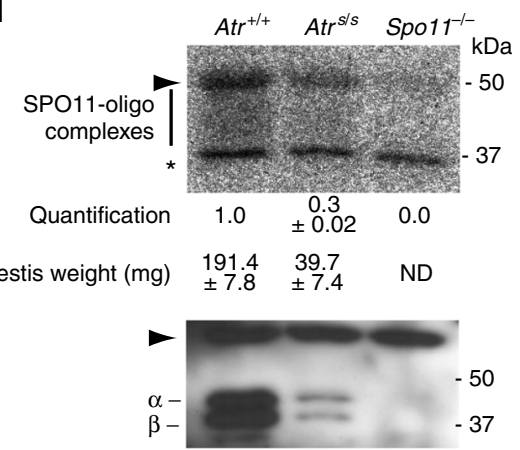

Modest recombination defects of Seckel mouse spermatocytes. $A_{t r} \mathrm{r}^{\mathrm{S} S}$ cells displayed subtle recombination defects. First, we detected persistent autosomal $\gamma \mathrm{H} 2 \mathrm{AX}$ patches in pachytene spermatocytes. During early prophase of meiosis, histone H2AX is phosphorylated by ATM in response to meiotic DSBs, which are repaired as prophase progresses $8,19,20$. In control mice, autosomal $\gamma \mathrm{H} 2 \mathrm{AX}$ progressively disappeared during prophase (Fig. 1d). From pachynema onward, $\gamma \mathrm{H} 2 \mathrm{AX}$ was mostly confined to the sex body, but a few $\gamma \mathrm{H} 2 \mathrm{AX}$ patches were visible on the autosomes, presumably at remaining unrepaired DSB sites. In $A t r / S$ spermatocytes, the numbers of autosomal $\gamma \mathrm{H} 2 \mathrm{AX}$ patches at early pachynema, late pachynema, and diplonema were higher than in wild type (Fig. 1e), suggesting delayed repair kinetics. Alternatively, Seckel spermatocytes might have an increased number of late-forming DSBs.

Second, whereas autosomal synapsis was unaffected, synapsis of sex chromosomes was impaired in $A t r^{S / S}$ cells (Supplementary Fig. 1c). Since the XY pair is almost completely heterologous except for the small pseudoautosomal region (PAR), sexchromosome synapsis must be mediated by DSBs occurring within the PAR ${ }^{21}$. Two-fold more $A t r^{S / S}$ spermatocytes displayed unsynapsed sex chromosomes at pachynema than control cells (12.2\% in $\mathrm{Atr}^{+/+}(N=197)$ vs. $25.1 \%$ in $\operatorname{Atr}^{S / S}(N=235), p=$ 0.0013 , Fisher's exact test). Atr ${ }^{+/ S}$ spermatocytes were similar to wild type ( $14.9 \%$ unsynapsed $\mathrm{X}$ and $\mathrm{Y}(N=131), p=0.2254$, Fisher's exact test).

To further investigate ATR function in meiotic recombination, we examined RAD51 and DMC1, which associate with resected DSBs and catalyze strand exchange ${ }^{22,23}$. We quantified these markers at progressive stages of meiotic prophase (Fig. 2a, b, Supplementary Table 1). In wild type, RAD51 and DMC1 foci were most abundant at leptonema, then progressively decreased through diplonema, when most foci localized to the nonhomologous portions of the sex chromosomes. RAD51 and $\mathrm{DMC1}$ focus numbers were higher in pachtyene $\mathrm{Atr}^{S / S}$ spermatocytes than in wild-type controls, consistent with the $\gamma \mathrm{H} 2 \mathrm{AX}$ results. In leptonema through zygonema, in contrast, fewer foci were observed in the mutant. Thus, there appear to be temporally distinct defects in recombination in the Atr ${ }^{S / S}$ spermatocytes.

The reduced focus counts in early spermatocytes could reflect either a deficiency in the formation or early processing of DSBs, or defective loading of RAD51 and DMC1. To distinguish between these possibilities, we examined early steps of DSB formation and processing. First, we studied whole-testis DSB levels in $\mathrm{Atr}^{\mathrm{S} / \mathrm{S}}$ mice

Fig. 2 Normal DSB formation but altered numbers of recombination markers in $A t r^{S / S}$ cells. a-c Left panels, quantification of total foci per spermatocyte for RAD51 (a), DMC1 (b), or RPA (c) at the indicated stages. Each point is the focus count for a single cell. Horizontal lines denote the mean. Right panels, representative images of spermatocytes stained for the indicated proteins. Scale bar $=10 \mu \mathrm{m}$. All $p$ values are from $t$ test pairwise comparisons; no $p$ value is stated if the comparison was not statistically significant ( $p>0.05$ ). d Quantification of SPO11-oligonucleotide complexes. A representative experiment is shown. Anti-SPO11 immunoprecipitates from two testes of each genotype were labeled with terminal transferase and $\left[\alpha-{ }^{32} \mathrm{P}\right]-\mathrm{dCTP}$, resolved by SDS-PAGE, and detected by autoradiography (top) and western blotting analysis (bottom). Vertical line indicates the signal from SPO11-oligonucleotide complexes; asterisk indicates non-specific signal from the labeling reaction; positions of the two major SPO11 splicing isoforms, $\alpha$ and $\beta$ are shown; and arrowheads denote the migration position of immunoglobulin heavy chain. SPO11-

oligonucleotide signal and testis weights are indicated (mean $\pm \mathrm{SD}, N=2$ ). Note that $A t r / S$ testes express both SPO11 $\alpha$ and SPO11 $\beta$, unlike mutants that experience arrested meiotic progression and lack SPO11 $\alpha$ as a consequence $^{37,63,64}$ 
by analyzing SPO11-oligonucleotide complexes, which are a direct readout of SPO11 function ${ }^{24}$. Unlike other mouse mutants with small testes ${ }^{25-27}, A t r S / S$ mice do not display prophase arrest, so the cellular composition (i.e., fractions of cell types) of their testes is similar to wild type (Supplementary Fig. 1b-d). Thus, to account for the dwarfism of Seckel mice ${ }^{14}$, we compared SPO11oligonucleotide signal in $A t r / S$ with wild type after normalization by testis weight. By this measure, we did not observe a reduction in SPO11 activity in $A t r / S$ testes; if anything, testis-weightnormalized SPO11-oligonucleotide levels were slightly elevated in mutant testes $(1.6 \pm 0.5$ fold, mean $\pm \mathrm{SD}, N=2)$ (Fig. $2 \mathrm{~d}$ ). These findings suggest that SPO11-induced DSB numbers are not reduced in $A t r / S$ spermatocytes.

Second, we examined the formation of RPA foci, which decorate ssDNA at resected DSB sites prior to the loading of RAD51 and $\mathrm{DMC1}^{22}$. In control spermatocytes, RPA focus numbers peaked in zygonema, then progressively decreased (Fig. 2c, Supplementary Table 1). RPA focus counts in Atr $S / S$ spermatocytes were elevated at early leptonema. A straightforward interpretation of the altered RPA, RAD51, and DMC1 focus numbers is that RPA tends to persist longer at DSB sites in Atr $\mathrm{S} / \mathrm{S}$ cells because of impaired loading of RAD51 and DMC1.

Physical linkages (chiasmata) between homologous chromosomes are crucial for accurate chromosome segregation at metaphase I. At least one crossover per bivalent is required to ensure proper orientation on the first meiotic spindle ${ }^{28}$. Because yeast Mecl participates in controlling the number and distribution of crossovers ${ }^{29,30}$, we investigated crossover formation in Seckel spermatocytes. In mice, several proteins have been involved in crossover formation, among them RNF212, which is the homolog of yeast Zip3. RNF212 has been implicated in designating CO sites by stabilizing key recombination proteins in a subset of recombination nodules ${ }^{31}$. Interestingly, $\mathrm{Atr}^{\mathrm{S} / \mathrm{S}}$ spermatocytes presented more RNF212 foci than control cells on average, and these foci tended to accumulate in clusters along stretches of the SCs (Fig. 3a, Supplementary Table 1).

We also examined MLH1, which is required for the formation of the majority ( $90 \%)$ of crossovers and which cytologically marks most crossover-designated sites at pachynema ${ }^{32}$. Pachytene $A t r S / S$ spermatocytes displayed one less MLH1 focus on average than controls (Fig. $3 \mathrm{~b}$ ), attributable to a significant increase in the number of autosomal bivalents lacking an MLH1 focus (Fig. 3c). However, we detected no difference in the number of achiasmate autosomal bivalents at diplonema (Fig. 3d). Overall, the RNF212 and MLH1 focus analysis may indicate that MLH1 focus formation is delayed in $A t r^{S / S}$ cells or that ATR regulates the number of crossovers that are repaired by MLH1dependent and -independent pathways.

Crossover control mechanisms regulate not only the number of crossovers but also their location ${ }^{33}$. The presence of one crossover inhibits the occurrence of another in its vicinity by a mechanism known as interference, which leads to a non-random distribution of crossovers $^{33,34}$. We asked whether ATR reduction affected the distribution of MLH1 foci. We found a similar distribution along autosomal SCs in $A t r S / S$ and wild type (Fig. 3e). In addition, the strength of cytological interference quantified using the gamma distribution $^{34}$ was similar in both genotypes (Fig. 3f). Thus, although wild-type ATR levels are required for proper MLH1 focus numbers at pachynema, focus distribution is not substantially altered in $A t r S / S$ cells.

ATR inhibition in vivo impairs meiotic recombination. Seckel mice do not completely lose Atr expression (Fig. 1a) ${ }^{14}$. Thus, to further assess the role of ATR in meiotic recombination, we established an experimental system to chemically inhibit ATR.
AZ20 is a selective ATR inhibitor that has been developed as a potential anti-cancer drug ${ }^{35}$. It inhibits ATR function in vitro and in cell culture systems, and its oral administration to mice carrying xenografted adenocarcinoma cells reduces tumor growth ${ }^{35}$

Wild-type adult males were dosed orally with AZ20 or vehicle solution (DMSO) for 3 days, the length of time normally required for a spermatocyte that initiates meiosis to complete homologous chromosome synapsis in vivo ${ }^{36}$. In control mice, $13.3 \%$ of testis cells were SYCP3-positive; of these, $81.5 \%$ were in pachynema or diplonema (Fig. 4a, b). AZ20-treated mice had fewer SYCP3-positive spermatocytes and altered proportions of prophase stages, including an increased fraction of zygotene cells $(p<0.0001$, Fisher's exact test) and an almost complete loss of diplotene cells ( $p<0.0001$, Fisher's exact test, see below) (Fig. 4a, b). We analyzed sex-body formation, judged by $\gamma \mathrm{H} 2 \mathrm{AX}$ deposition on the $\mathrm{X}$ and $\mathrm{Y}$ chromatin of pachytene cells, as a readout of ATR function. Compared with controls, AZ20-treated mice had 13-fold more pachytene cells lacking a sex body (DMSO: $0.25 \pm 0.35 \%, N=400 ; \mathrm{AZ20}$ : $3.25 \pm 0.35 \%, N=400$; Mean \pm SD, $p=0.014$, one-way ANOVA, Fig. $4 d$ ). These data indicate that in vivo treatment with AZ20 inhibits ATR activity in meiosis and that this inhibition affects meiotic progression.

Next, we analyzed sex-chromosome synapsis and recombination markers. Similar to results with $A t r{ }^{S / S}$ mice, AZ20 treatment caused a more than two-fold increase in the frequency of unsynapsed sex chromosomes at pachynema (Fig. 4c). AZ20treated spermatocytes exhibited fewer RAD51 foci during leptonema and early zygonema, and, as observed in $A t r S / S$ spermatocytes, the decrease in RAD51 focus number was accompanied by a significant increase in the number of RPA foci at early leptonema (Fig. 4e, Supplementary Table 2). These results suggest that RAD51 association with resected DSBs is delayed in spermatocytes from AZ20-treated males, which could in turn affect sex chromosome synapsis.

To corroborate the ATR role in the dynamics of late recombination foci, we treated adult males with AZ20 or DMSO for 7 days, the time required for a leptotene spermatocyte to reach $\mathrm{mid} /$ late-pachynema. This longer treatment resulted in a slightly reduced percentage of SYCP3-positive spermatocytes and an increased number of apoptotic cells per tubule (Fig. 4a, b, and f). The prolonged treatment resulted similarly in a large increase in the fraction of pachytene spermatocytes lacking a sex body (DMSO: $0.5 \pm 0.7 \%, N=200 ;$ AZ20-treated: $14.5 \pm 0.7 \%, N=$ 200, Mean \pm SD, $p=0.0002$, one-way ANOVA). Importantly, treated mice lacked diplotene spermatocytes (Fig. 4b), in agreement with a previous report that ATR function is required to exit pachynema ${ }^{5}$. Similar to Seckel mice, AZ20-treated animals accumulated more RAD51 foci at pachynema, corroborating the requirement of ATR for timely repair of DSBs (Fig. 4e, Supplementary Table 2). In addition, AZ20-treated mice accumulated RNF212 at early and late pachynema (Fig. 4g, Supplementary Table 2) and displayed, on average, one less MLH1 focus than controls (Fig. 4h). The lack of diplotene spermatocytes in AZ20-treated mice precluded study of achiasmate bivalents. As in $A t r S / S$ spermatocytes, the distribution of MLH1 foci was not substantially altered in AZ20-treated animals and MLH1 interfocal distances displayed positive interference (Fig. 4i, j). Furthermore, modeling interfocal distances to a gamma distribution gave lower values of cytological interference than the controls, but within the range considered normal in other studies ${ }^{20,34}$.

In sum, the results from in vivo treatment of wild-type mice recapitulated our findings with the Seckel mouse model, with some differences that can be attributed to a greater decrease in ATR function from AZ20 treatment. Specifically, unlike Atr $\mathrm{S} / \mathrm{S}$ mice, AZ20-treated mice displayed meiotic prophase arrest and 
more severe alterations to focus counts for recombination markers (Supplementary Tables 1 and 2). Also, whereas almost all pachytene $A t r^{S / S}$ spermatocytes displayed a sex body, $15 \%$ of pachytene spermatocytes from mice treated 7 days with AZ20 did not. Thus, reduced ATR activity may prevent sex-body formation, which would cause pachytene arrest and an increase in apoptosis. Moreover, we showed previously that abnormal accumulation of ATR on the XY pair may be sufficient to phosphorylate H2AX but insufficient to implement silencing ${ }^{37}$. Thus, we postulate that the arrest observed after in vivo treatment with AZ20 may be the consequence of a failure to silence the sex chromosomes at pachynema.

In vitro ATR inhibition impairs meiotic prophase progression. Although AZ20 treatment in vivo appeared to attenuate ATR activity more than the Seckel mutation did, the accumulation of $\gamma \mathrm{H} 2 \mathrm{AX}$ on the XY chromatin in most treated spermatocytes indicated that ATR function was not fully blocked. This might be attributable to the blood-testis barrier, which controls entry of

a
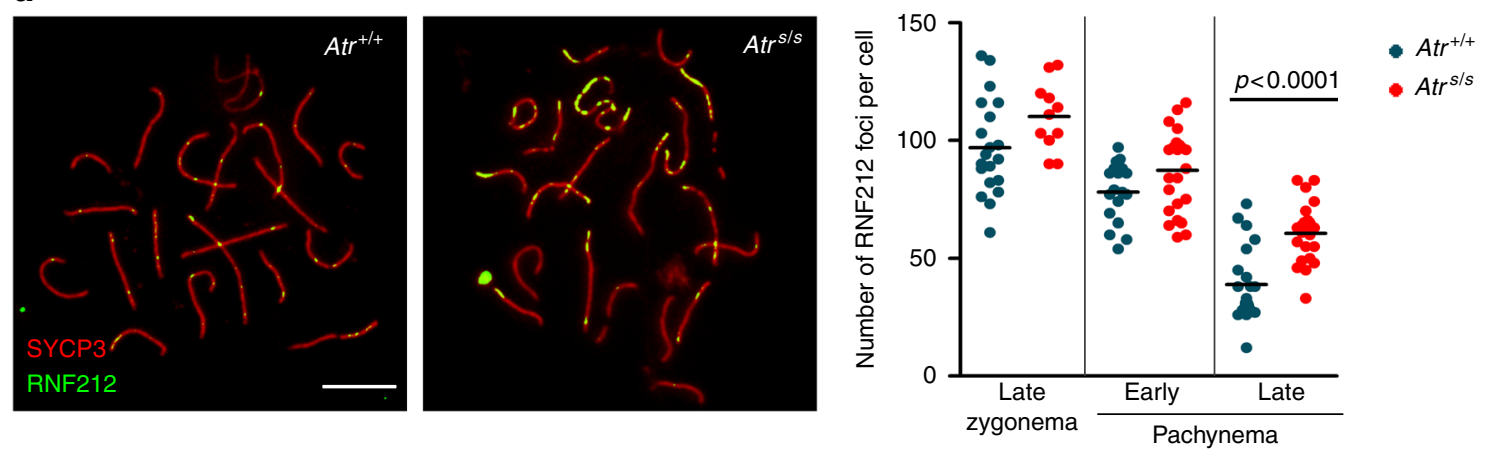

b
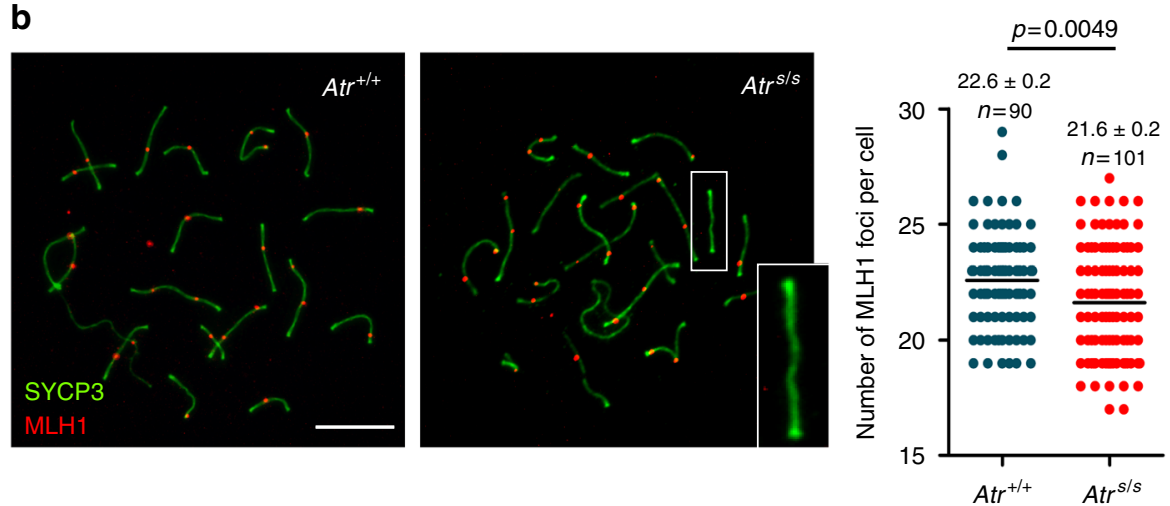

C

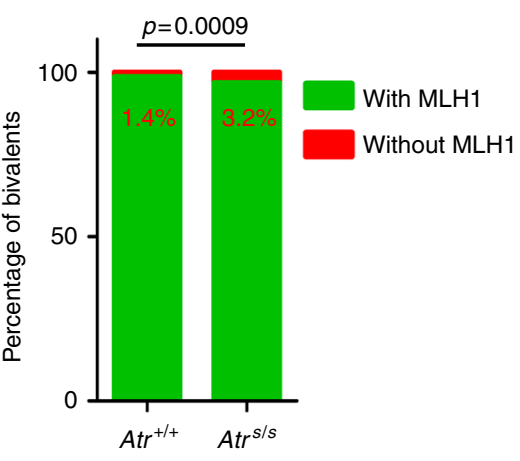

d
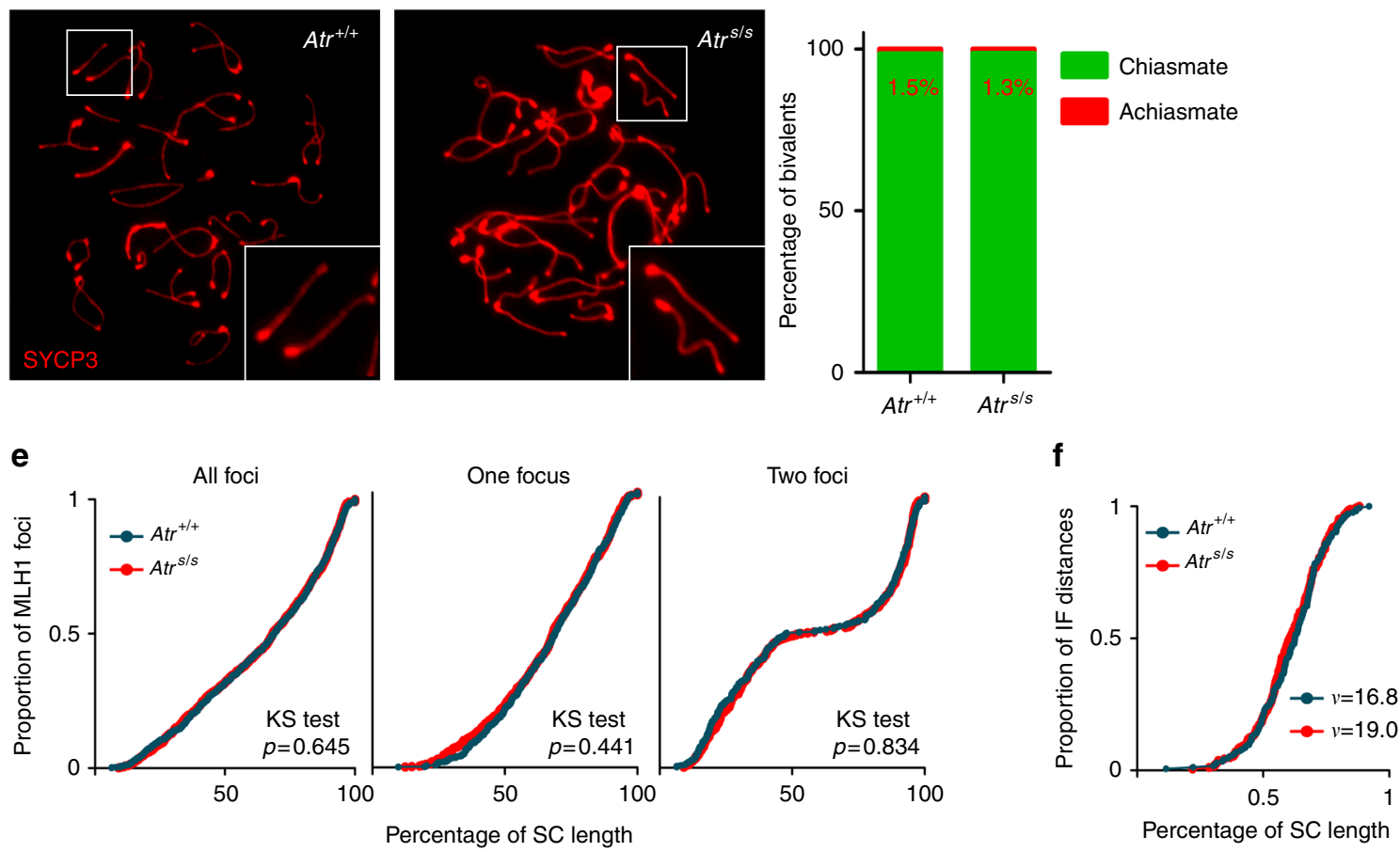
molecules from circulating blood into the seminiferous tubules ${ }^{38}$. Thus, we cultured fragments of neonatal testes in media containing AZ20. We hypothesized that this strategy could surmount the blood-testis barrier, thereby increasing exposure of spermatocytes to AZ20 and better inhibiting ATR function.

We cultured testis fragments isolated from 5 days post partum (dpp) wild-type mice. Because ATR inhibition can interfere with DNA replication ${ }^{39}$, fragments were initially cultured without treatment for 7 days to allow entry into meiosis. Then, for a further 7 days, fragments were cultured either without treatment or in the presence of different doses of $\operatorname{AZ20}(0.2,1$, or $5 \mu \mathrm{M})$ or equivalent volumes of DMSO (DMSO1, DMSO2, or DMSO3).

We used SYCP3 to assess meiotic prophase initiation, progression, and synapsis. At day zero (D0, i.e., prior to culture), only $1 \%$ of testis cells were SYCP3-positive and were thus judged to be spermatocytes (Fig. 5a, b). After the initial 7 days of culture (D7), $13 \%$ of testis cells were spermatocytes, of which $75 \%$ were at leptonema. After 2 weeks of culture (D14), 16\% of testis cells from untreated samples showed SYCP3 staining. Importantly, $25 \%$ of these D14 spermatocytes displayed complete synapsis consistent with having reached pachynema. A low dose of AZ20 had no effect on meiotic progression: at D14, proportions of spermatocytes at different prophase stages were indistinguishable between $0.2 \mu \mathrm{M}$ AZ20-treated, DMSO1-treated, and untreated samples (Fig. 5a-d). However, at higher doses (1 or $5 \mu \mathrm{M})$, cultured samples exhibited a decreased fraction of SYCP3positive cells compared with DMSO-treated controls, suggesting that some spermatocytes had died between D7 and D14. We did not detect any spermatocytes with complete synapsis or with a sex body (Fig. 5c-e, Supplementary Table 3).

We used $5 \mu \mathrm{M}$ AZ20-treated samples to evaluate whether recombination defects observed in the in vivo models were recapitulated in vitro. Whereas untreated and DMSO3 samples exhibited similar RAD51 focus dynamics, dramatically fewer foci were observed in $5 \mu \mathrm{M}$ AZ20-treated samples (Fig. 5f, g, Supplementary Table 4). A severe effect on DMC1 focus formation was likewise seen (Fig. 5h). Similar to the in vivo models, RPA focus counts at early leptonema were higher from 5 $\mu \mathrm{M}$ AZ20-treated samples than in controls (Fig. 5i, Supplementary Table 4). DMSO treatment alone appeared to cause modest changes, as fewer RPA foci were observed in late leptotene and late zygotene spermatocytes in DMSO3 samples compared with untreated, possibly due to DMSO toxicity (Fig. 5i). Nonetheless, AZ20-treated samples behaved differently from DMSO3-treated samples at all prophase stages. These data show that inhibition of ATR function alters RAD51 and DMC1 loading.

CHK1 function is required for proper RAD51 loading. Checkpoint kinase 1 (CHK1) is the major effector of the ATR signaling pathway ${ }^{3}$. In somatic cells ATR activates CHK1, which interacts with and phosphorylates RAD51 to promote repair of DNA lesions ${ }^{3,40}$. To investigate the effect of CHK1 perturbation on meiotic recombination, we targeted CHK1 protein in vitro with two specific inhibitors, PF- $477736^{41}$ and LY2603618 ${ }^{42}$ (referred to as CHK1i). As above, we cultured $5 \mathrm{dpp}$ testis fragments for 7 days before subjecting them to CHK1i for an additional 7 days.

As assessed by SYCP3 immunostaining at D14, CHK1i-treated samples displayed fewer SYCP3-positive spermatocytes than DMSO-treated controls and no spermatocytes that had completed synapsis (Fig. 5c). Moreover, RAD51 focus numbers were substantially reduced, indicating that RAD51 loading onto chromosome axes was severely compromised (Fig. 5j, k). We conclude that disruption of the ATR-CHK1 pathway affects loading of RAD51 onto resected DSBs and restricts progression through meiotic prophase.

ATR promotes SC elongation. The absence of spermatocytes with complete SC formation after in vitro inhibition of ATR or CHK1 (Fig. 5c-e, Supplementary Table 3) suggested that ATR may participate in SC formation and/or elongation. We used a Spo11 mutant to study how in vivo inhibition of ATR affected SC formation and/or elongation in the absence of meiotic recombination $^{43}$. Spo11 $1^{-/-}$spermatocytes fail to form DSBs but still form stretches of SC, so SC formation is independent of meiotic recombination in this genetic background. AZ20-treated Spo11 $11^{-/}$ spermatocytes displayed the same number of SC stretches per cell as untreated Spo11 $11^{-1-}$ cells (Supplementary Fig. 2a, b). However, the average length of these fragments was shorter in AZ20-treated spermatocytes (Supplementary Fig. 2a, c). These findings suggest that wild-type levels of ATR function are not required for SC initiation but play an important role in SC elongation.

Accumulation of recombination markers on unsynapsed axes. In yeast, Mecl regulates meiotic recombination partner choice by phosphorylating Hop $1^{44,45}$, the ortholog of mouse HORMA domain-containing protein HORMAD1. HORMAD1 is involved in several meiotic processes, localizes to chromosome axes at the onset of meiosis and becomes phosphorylated during meiotic progression $^{46,47}$ (discussed further below). In mouse, unsynapsed chromosomes tend to accumulate recombination markers during zygonema $^{48-51}$, implying that DSBs are continuously formed and processed in unsynapsed regions until the homolog is engaged for repair. Because ATR decorates unsynapsed axes in mice ${ }^{5}$, where HORMAD1 accumulates, we asked whether ATR plays a role in DSB formation and early processing specifically in unsynapsed regions.

The X chromosome normally remains completely unsynapsed throughout zygonema ${ }^{40}$, so it is a useful model for examining the kinetics of recombination markers on unsynapsed axes ${ }^{48}$. We

\footnotetext{
Fig. 3 Late recombination markers in wild type and $A t r / S$ spermatocytes. a Left panels, representative images of late pachytene spermatocytes immunostained against SYCP3 and RNF212. Scale bar $=10 \mu \mathrm{m}$ and applies to both images. Right panel, quantification of RNF212 foci. Horizontal black lines denote the means; the $p$ value is from $t$ test, and comparisons without $p$ values stated were not significant $(p>0.05)$. $\mathbf{b}$ Left panels, representative images of pachytene spermatocytes immunostained against SYCP3 and MLH1. Scale bar $=10 \mu \mathrm{m}$ and applies to both images. A bivalent lacking an MLH1 focus in the $A t \mathrm{~S}^{\mathrm{S} / \mathrm{S}}$ spermatocyte (white box) is magnified in the inset. Right panel, quantification of autosomal MLH1 foci at pachynema. Horizontal black lines denote the means. Atr $\mathrm{S} / \mathrm{S}$ and control were compared by Mann-Whitney test. c Proportion of bivalents without an MLH1 focus. $N=1520$ (mutant) or 1653 (control); $p$ value is from Fisher's exact test. $\mathbf{d}$ Left, representative images of diplotene spermatocytes immunostained against SYCP3. Scale bar $=10 \mu \mathrm{m}$. Achiasmate bivalents (white boxes) are magnified in the insets. Right, percentage of achiasmate bivalents at late diplonema from $A t r+/+(N=798)$ and $\operatorname{Atr}^{\mathrm{S} / \mathrm{S}}(\mathrm{N}=912)$ spermatocytes ( $p=0.84$, Fisher's exact test). e Cumulative frequency plots comparing MLH1 focus distribution along autosomal bivalents from pachytene $A t r^{+/+}$and $A t r^{S / S}$ spermatocytes. MLH1 focus distributions were similar between $A t r+/+(N=924)$ and $A t r S / S(N=932)$. Distributions in SCs presenting one $\mathrm{MLH} 1$ focus from $\operatorname{Atr}^{+/+}(N=581)$ and $A t r S / S(N=601)$ and in SCs presenting two MLH1 foci from $A t r+/+(N=350)$ and $A t r / S(N=$ 369) spermatocytes were also indistinguishable (Kolgomorov-Smirnov (KS) test). f MLH1 interfocal distances (as a percentage of SC length) measured on SCs containing more than one MLH1 focus. Data were fitted to the gamma distribution to measure the strength of interference denoted by the shape parameter $(\nu)^{34}$
} 
scored RAD51 and RPA focus density on the X chromosome at early and late zygonema in wild-type and $A t r S / S$ spermatocytes (Fig. 6a-d). The densities of RAD51 and RPA foci in wild type were higher in late zygonema than early zygonema $(p=0.0005$ for RAD51 and $p=0.023$ for RPA, $t$ test) (Fig. 6a-d), as previously reported ${ }^{48}$. In $A \mathrm{tr}^{S / S}$ spermatocytes in contrast, neither RAD51 focus density, which was substantially lower at both substages, nor RPA focus density rose significantly as zygonema progressed ( $p>0.05, t$ test) (Fig. 6a-d).
To corroborate these findings, we asked whether culturing testis fragments in $5 \mu \mathrm{M}$ AZ20 also curtailed accumulation of recombination markers on unsynapsed chromosomes (Fig. 6e). Indeed, AZ20-treated samples exhibited a significant decrease in the density of RPA foci on unsynapsed axes as zygonema progressed ( $p=0.0004, t$ test). Similar to our earlier observations for total RPA counts, DMSO treatment affected RPA focus density on unsynapsed axes, but AZ20 treatment further reduced this density. These findings indicate that ATR contributes to the a

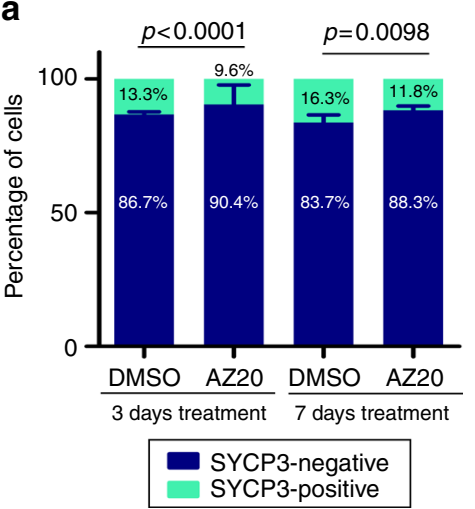

e

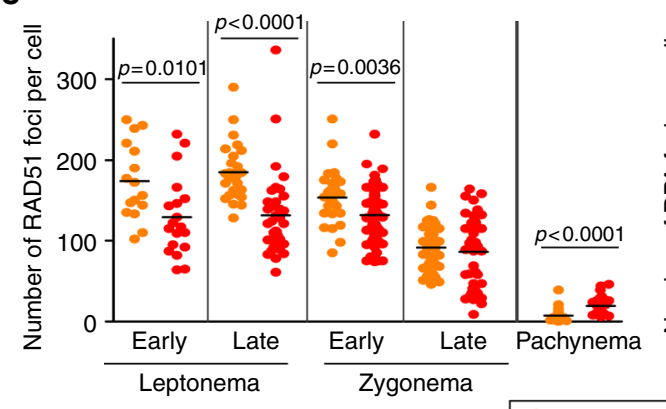

b

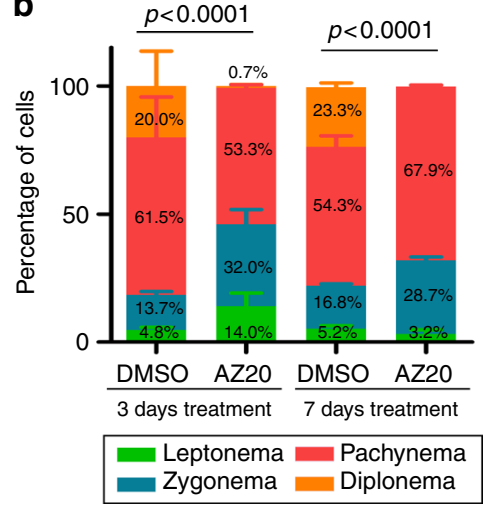

C

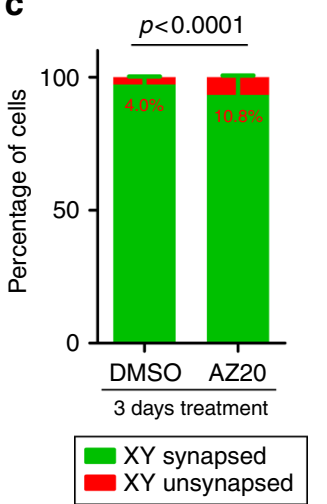

d

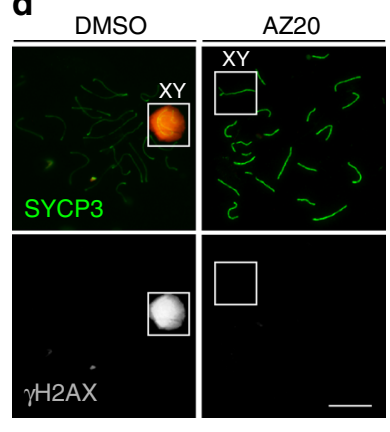

$\mathbf{f}$

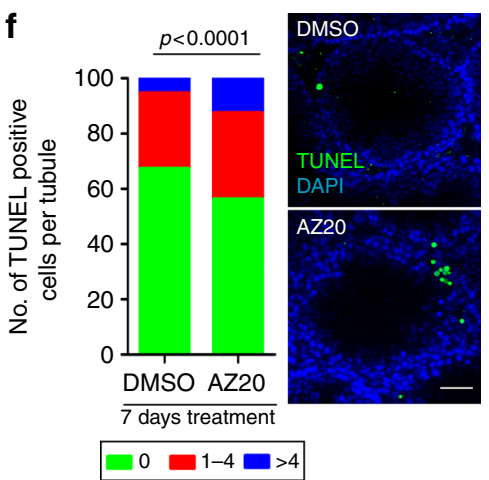

g

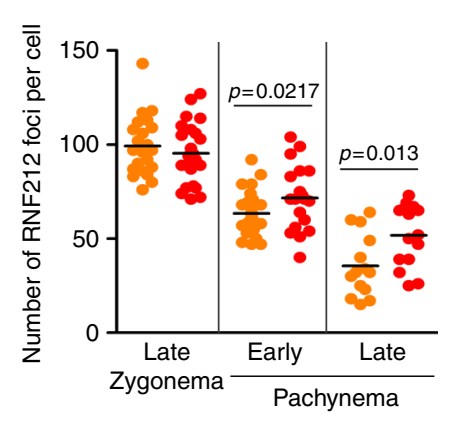

h

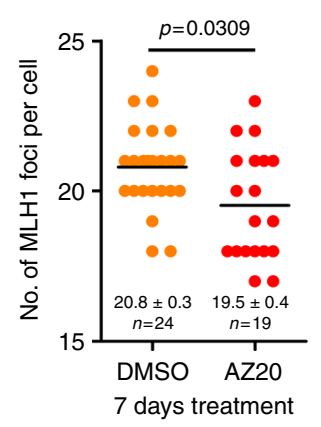

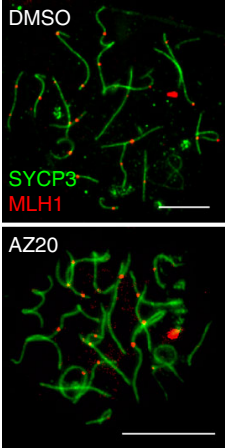

i
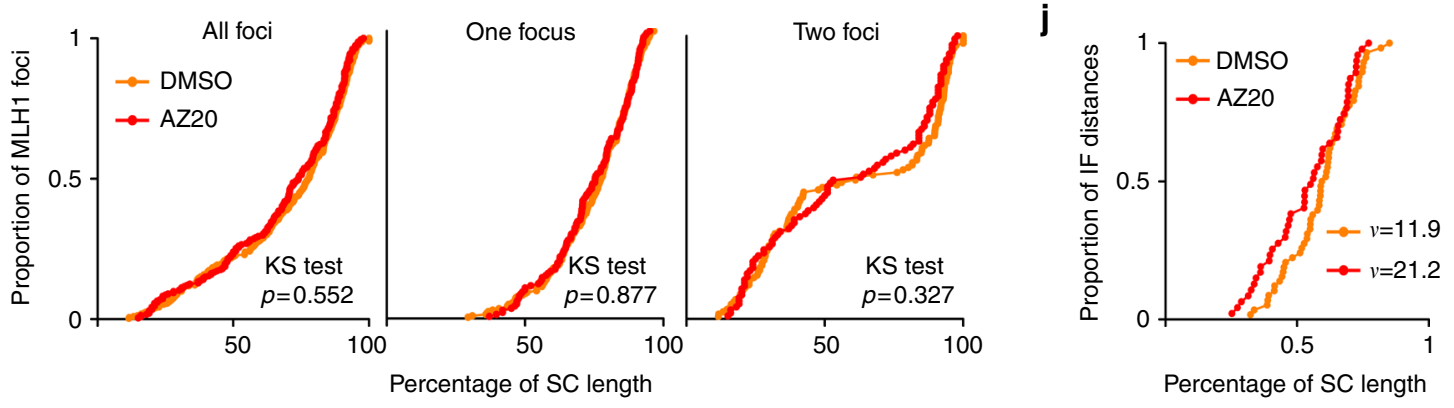
normal patterns of accumulation of recombination markers on unsynapsed chromosome axes.

\section{Discussion}

In this study, we used a combination of genetic and pharmacological tools to examine the function of ATR in meiotic recombination. This strategy is a useful approach for studying the meiotic role(s) of a protein whose null mutation is embryonically lethal. Although conditional models can also address this issue, the use of specific inhibitors is advantageous because mice of desired genotypes are more readily obtained when studying a single locus. This aspect significantly reduces the number of mice required to perform the study.

An accompanying study using conditional Atr mutant mice corroborates our results by reporting the role of ATR in promoting homologous chromosome synapsis, SC elongation, RAD51 and DMC1 loading onto DSBs, and maintenance of recombination foci on unsynapsed axes ${ }^{52}$. However, this mutant mouse displays meiotic arrest at mid pachynema that prevents studying ATR functions past this point. In this sense, the use of a hypomorphic mutation and a pharmacological strategy has proven effective to investigate ATR functions in some meiotic processes, like crossover formation and distribution, that are obscured by arrest of prophase progression. Thus, these two strategies complement one another.

Using our approach, ATR function could be inhibited to different degrees, as determined by meiotic prophase progression and sex-body formation. ATR is known to be required to form the sex body ${ }^{5}$. Upon in vitro administration of AZ20, spermatocytes were blocked by zygonema (i.e., prior to sex-body formation), so we infer that this condition delivered the strongest inhibition. In vivo administration of AZ20 allowed accumulation of pachytene spermatocytes, thus inhibition was not as strong as in the in vitro setting. Finally, $A t r^{S / S}$ spermatocytes displayed grossly normal meiotic progression and nearly wild-type levels of sex-body formation. Of note, markers of other meiotic processes (e.g., RAD51 focus counts and XY synapsis) were also more perturbed in AZ20-treated samples than in $\mathrm{Atr}^{\mathrm{S} / \mathrm{S}}$ mice.

Our data show that ATR and its effector CHK1 play a critical role in the loading of RAD51 and DMC1 onto chromatin. While RAD51 focus numbers were reduced in $\mathrm{Atr}^{S / S}$ spermatocytes relative to wild type only at early leptonema, DMC1 focus numbers were significantly lower from early leptonema through late zygonema. As expected from previous studies performed in mammals and other organisms ${ }^{53,54}$, the different effects on the kinetics of RAD51 and DMC1 foci corroborate that these recombinases are regulated differently. Distinct regulation may be attributable to several factors. First, while the protein sequences of mouse RAD51 and DMC1 are very similar (53.3\% identical and $64 \%$ similar), they differ at a residue that may be critical for RAD51 activity. In mammalian somatic cells, RAD51 Threonine 309 is phosphorylated by CHK1 upon activation of ATR by RPAcoated DNA structures, and this post-translational modification promotes RAD51 focus assembly ${ }^{40}$. It has been suggested that CHK1-dependent RAD51 phosphorylation is required for RAD51 to displace RPA from resected DSB ends and initiate homology search ${ }^{40}$. The paralogous residue in DMC1 is Leucine 310 , hence it cannot be similarly regulated. Second, the current favored model of RAD51 and DMC1 loading onto resected DSBs in meiosis proposes that RAD51 loading is a prerequisite for the incorporation of $\mathrm{DMC}^{53}$. Thus, this interdependency could also account for the more severe effect on DMC1 focus formation observed in ATR-defective spermatocytes.

Aberrant loading and kinetics of RAD51 and DMC1 may have implications for homologous chromosome pairing and synapsis. Cytologically, the phenotype of ATR-deficient spermatocytes (altered kinetics of recombination markers, reduced number of MLH1 foci at pachynema, increased proportion of spermatocytes at zygonema, and defects in sex-chromosome synapsis) is consistent with impaired meiotic recombination. However, our data in the DSB-deficient Spo11 $1^{-/}$background indicate that ATR can also promote SC extension independently of its role in meiotic recombination. Several proteins of the chromosome axis, including HORMAD1, are phosphorylated during meiotic prophase $^{55}$. HORMAD1 is required for normal numbers of meiotic DSBs, proper SC formation and recruitment of ATR onto unsynapsed chromosome axes ${ }^{47}$. At the onset of meiotic prophase HORMAD1 is localized at the chromosome $\mathrm{axes}^{46}$. As a result of DSB formation, HORMAD1 is phosphorylated, presumably by $\mathrm{ATR}^{5,55}$, and when homologous chromosomes synapse, HORMAD1 is displaced from the axes. Thus, we hypothesize that the role of ATR in SC elongation may be mediated by HORMAD1. Indeed, the yeast HORMAD1 ortholog Hop1 is phosphorylated by Mec1 and Tel1 (the orthologs of ATR and ATM), and this phosphorylation promotes SC formation ${ }^{44}$.

In budding yeast, Mecl participates in two additional key aspects of meiotic recombination. First, it biases recombination toward the homologous chromosome over the sister chromatid as the preferred template for meiotic DSB repair ${ }^{45}$. Second, it indirectly promotes DSB formation during meiotic prophase by inhibiting the transcription factor Ndt80, which is required to exit pachynema and thereby close the cell cycle window during which Spo11 forms DSBs ${ }^{56}$. In yeast, inter-sister meiotic recombination is over three times faster than inter-homolog recombination ${ }^{44}$. If ATR participates similarly in the implementation of inter-

Fig. 4 In vivo inhibition of ATR affects prophase progression and recombination markers. a Percentage of SYCP3-positive cells from mouse testes treated 3 or 7 days with DMSO $(N=2057$ and $N=986$ cells, respectively) or AZ20 ( $N=2063$ and 936 cells, respectively). Two mice per condition were analyzed. $P$ value is from Fisher's exact test. $\mathbf{b}$ Percentage of spermatocytes at different prophase stages in 3 and 7 days DMSO- $(N=2035$ and $N=600)$ and AZ2Otreated mice $(N=2091$ and $N=600$ cells, respectively). Two mice per condition were analyzed. $P$ value is from $G$ test. c Percentage of pachytene cells exhibiting unsynapsed $X$ and $Y$ chromosomes in DMSO- $(N=472)$ and AZ2O-treated spermatocytes $(N=426)$. Two mice per condition were analyzed. $P$ value is from Fisher's exact test. d Representative images of DMSO- and AZ2O-treated pachytene spermatocytes stained for SYCP3 and $\gamma \mathrm{H} 2 \mathrm{AX}$. Note the presence of a sex body over the $X$ and $Y$ chromosomes in the control, but not in the AZ20-treated cell. e RAD51 and RPA foci per spermatocyte at the indicated stages in DMSO- and AZ20-treated mice. Horizontal lines denote the means. $P$ values are from $t$ tests. f Proportion of tubule sections with 0 , 1-4, or $>4$ TUNEL-positive cells from mice treated with DMSO or AZ2O. $P$ value is from $t$ test. Scale bar $=40 \mu \mathrm{m}$. $\mathbf{g}$ RNF212 foci in pachytene spermatocytes after 7 days of DMSO or AZ2O treatment. Horizontal black lines denote the means. $P$ value is from $t$ test. Images show pachytene spermatocytes immunostained for SYCP3 and RNF212. Scale bars $=10 \mu \mathrm{m}$. h Autosomal MLH1 foci in pachytene spermatocytes after 7 days of DMSO or AZ20 treatment. Horizontal black lines denote the means. $P$ value is from a Mann-Whitney test. Images show pachytene spermatocytes immunostained for SYCP3 and MLH1. Scale bars $=10 \mu \mathrm{m}$. i Cumulative frequency plots comparing MLH1 focus distribution along autosomal bivalents from pachytene spermatocytes after 7 days of DMSO or AZ20 treatment. MLH1 focus distribution along all autosomal bivalents was similar between DMSO

$(\mathrm{N}=320)$ and $\mathrm{AZ2O}(\mathrm{N}=233)$ treatments. In SCs presenting one MLH1 focus from DMSO- $(N=204)$ and $\mathrm{AZ20}-(N=138)$ treated mice or two MLH1 foci from DMSO- $(N=116)$ and AZ2O- $(N=94)$ treated mice, MLH1 focus location was also indistinguishable (KS test). $\mathbf{j}$ MLH1 interfocal distances expressed as a percentage of SC length from autosomal bivalents containing two MLH1 foci. Shape parameters $(\nu)$ from gamma distribution are shown ${ }^{34}$ 
homolog bias in mouse, ATR-deficient spermatocytes might repair DSBs more rapidly by recombination between sister chromatids. This model could explain the observed reduction in recombination marker density on unsynapsed axes at zygonema. In support of this model, the reduction in RPA foci at zygonema in $A t r / S$ spermatocytes despite globally unaltered DSB numbers could reflect a more rapid turnover of recombination markers by inter-sister repair. However, our data also provide results that seem inconsistent with this interpretation. The increase in recombination markers $(\gamma \mathrm{H} 2 \mathrm{AX}, \mathrm{RAD} 51$, and DMC1) on the synapsed axes of pachytene-stage $A t^{S / S}$ spermatocytes appears difficult to reconcile with faster repair by inter-sister recombination. However, it is also plausible that ATR may play another role in DSB repair from mid/late pachynema onward. It has been a

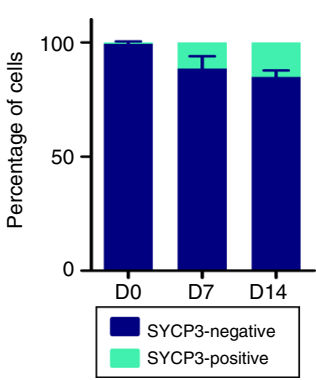

b
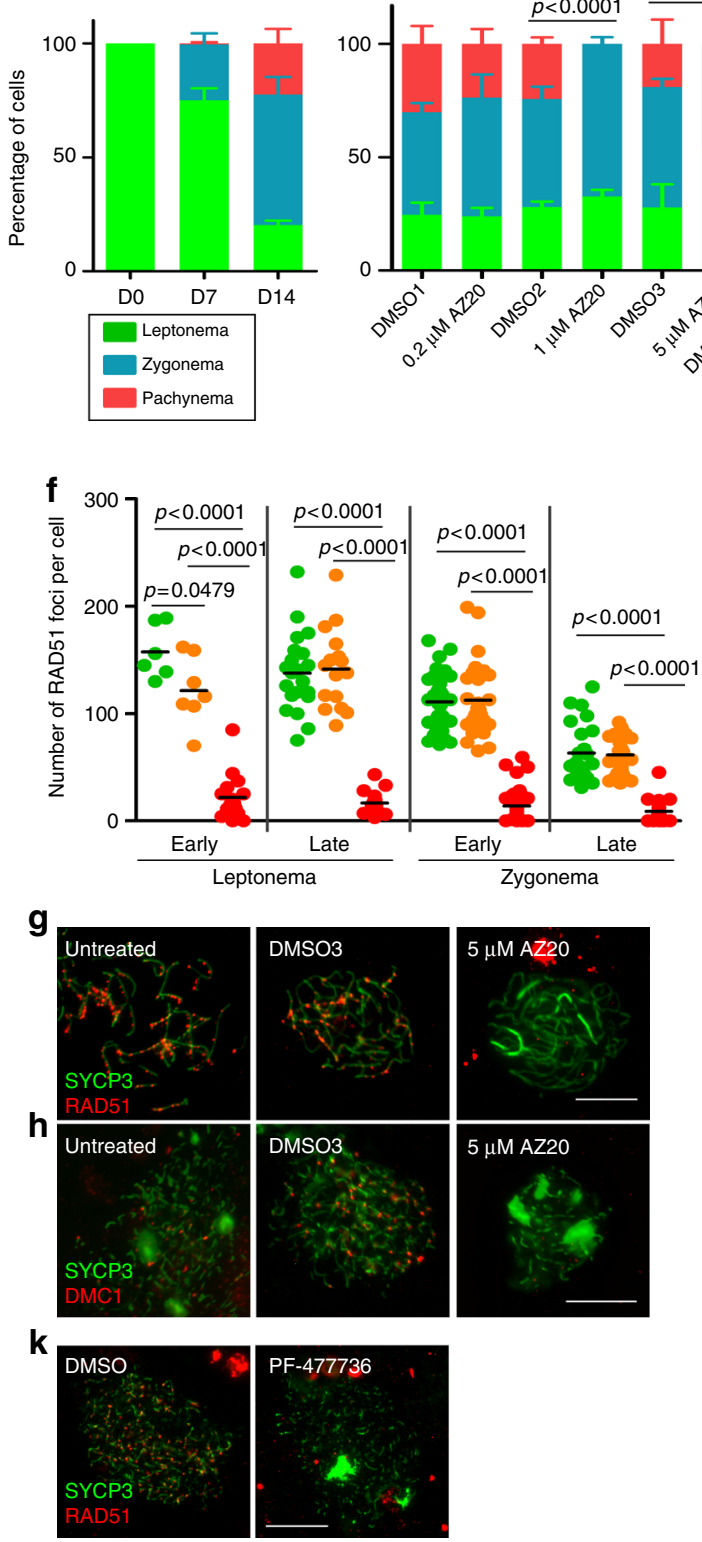

C

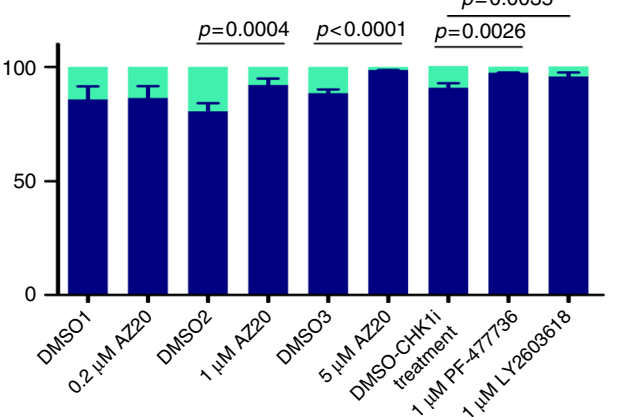

d

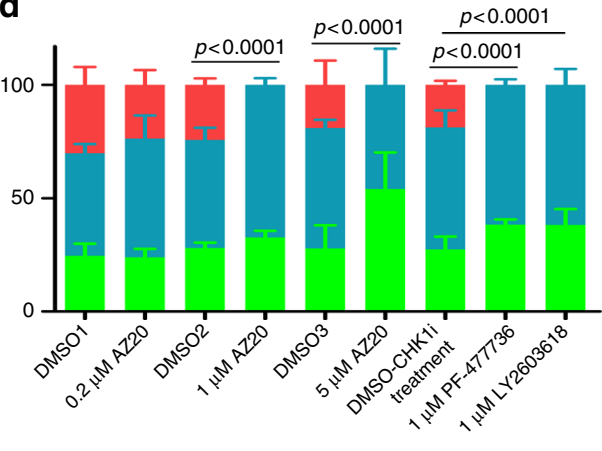

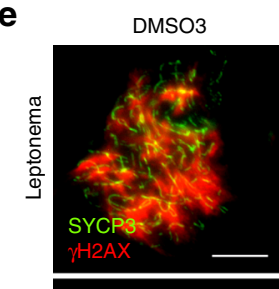
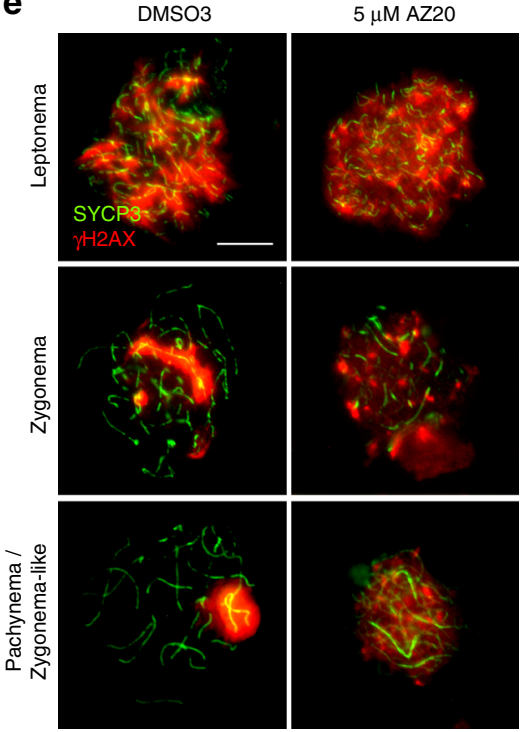
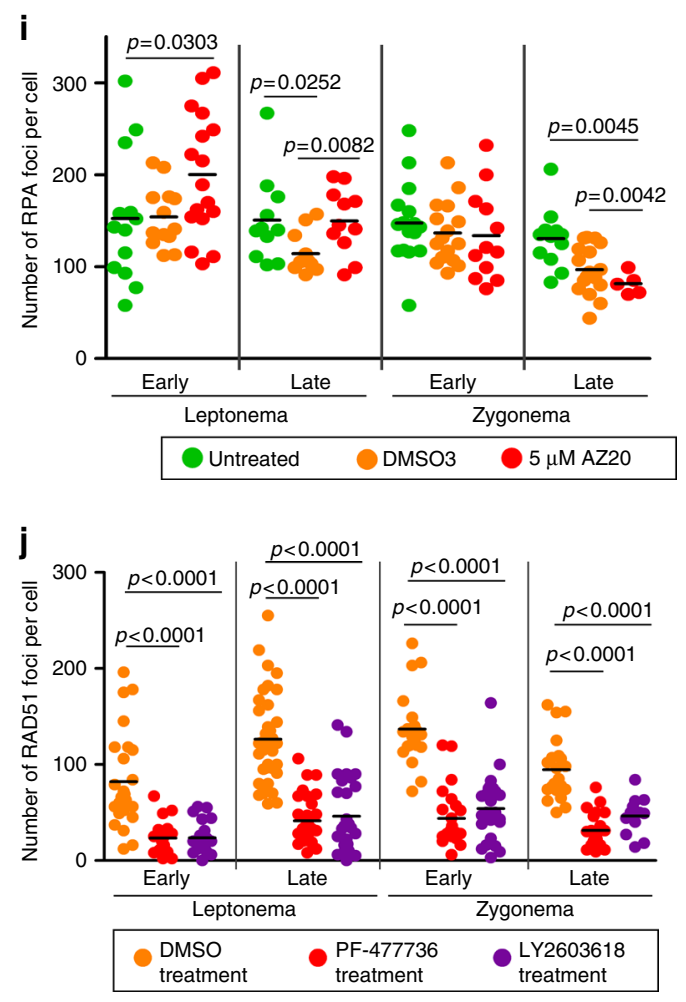
proposed that DSB repair processes other than canonical meiotic recombination may be active from mid pachynema onward ${ }^{19}$, including non-homologous end joining (NHEJ) ${ }^{57}$. Spermatocytes from mice deficient for KU70, which is required for NHEJ, present more $\gamma \mathrm{H} 2 \mathrm{AX}$ patches at late pachynema and diplonema than control cells, suggesting that NHEJ participates in DSB repair at late meiotic prophase ${ }^{59}$. Thus, the accumulation of $\gamma \mathrm{H} 2 \mathrm{AX}$ patches observed at mid/late pachynema and diplonema in Seckel mice could also reflect that ATR is required to activate alternative DSB repair pathway(s) at later stages of meiotic prophase.

Finally, the presence of normal numbers of achiasmate bivalents at diplonema in Seckel mouse spermatocytes suggests that ATR function in that context is sufficient for normal levels of crossover formation. We did not observe enough in vivo AZ20treated diplotene spermatocytes to study chiasma formation when ATR function was further reduced. Nonetheless, our data show that ATR function is required for the proper number of MLH1 foci at pachynema.

In summary, our data show that the ATR-CHK1 pathway is required for the completion of meiotic recombination in mammalian spermatocytes. RAD51 and DMC1 kinetics are altered when ATR or CHK1 function is attenuated, suggesting a role for this pathway in loading RAD51 and DMC1 onto resected DSBs. Furthermore, deficiency in ATR function affects SC formation and the accumulation of recombination markers along unsynapsed axes during zygonema. Thus, reduction in ATR function may impair the ability of homologous chromosomes to pair and synapse during meiotic prophase. Finally, our finding that in vivo exposure to AZ20 causes meiotic prophase arrest is relevant when considering the use of ATR inhibitors in cancer therapy, since meiotic arrest may result in infertility. Further studies should be performed to determine how long this arrest lasts after exposure to the drug.

\footnotetext{
Methods

Mice and genotyping. The Atr Seckel $\left(\mathrm{Atr}^{S}\right)$ and Spo11 alleles were generated previously ${ }^{14,43}$. Genotyping was performed by PCR of tail tip DNA using previously designed primers ${ }^{14,43}$. The $\mathrm{Atr}^{S}$ allele was maintained on a C57BV6-129/Sv mixed genetic background. Experiments were performed using two or more mutant or treated animals and compared with littermate control mice. When appropriate littermate controls were unavailable, control animals were obtained from other litters of the same matings and at the same age. All animals were killed using $\mathrm{CO}_{2}$ euthanization methods. Experimental procedures conform to the protocol CEEAH 1091 (DAAM6395) approved by the Ethics Committee for Animal Experimentation of the Universitat Autònoma de Barcelona and the Catalan Government.
}

RNA expression analysis. RNA from mouse testes was extracted using the RNeasy Plus Mini kit (Qiagen). The SuperScript III One-Step RT-PCR Taq High Fidelity (Invitrogen) was used to synthesize cDNA and amplify Atr transcripts using previously designed primers ${ }^{16}$

Cytology, immunostaining, and FISH. Testes were dissected from mice at 2-3 months of age and were processed for cytology and histology, as previously described ${ }^{50,58}$. Briefly, for surface spreading procedures, the tunica albuginea was removed and seminiferous tubules were released and minced to obtain a single-cell suspension. Cells were treated with a hypotonic solution $(0.1 \mathrm{M}$ sucrose $)$ and fixed in $1 \%$ paraformaldehyde in $\mathrm{H}_{2} \mathrm{O}$ with $0.1 \%$ Triton $\mathrm{X}-100$ for $2 \mathrm{~h}$. Slides were washed in $0.4 \%$ Kodak Photo-Flo and air-dried. Immunostaining of surface spreads was performed using standard methods ${ }^{37}$. The following primary antibodies were used: rabbit anti-SYCP3 (Abcam) 1:200 dilution, mouse anti-SYCP3 (Abcam) 1:200 dilution, mouse anti-SYCP1 (Abcam) 1:400 dilution, mouse anti- $\gamma \mathrm{H} 2 \mathrm{AX}$ (Millipore) 1:200 dilution, guinea pig anti-H1T (kindly donated by Dr. Mary Ann Handel) 1:500 dilution, rabbit anti-ATR (Calbiochem) 1:100 dilution, mouse antiSUMO-1 (Life Technologies) 1:100 dilution, sheep anti-MDC1 (AbdSerotech) 1:100 dilution, rabbit anti-RAD51 (Calbiochem) 1:100 dilution, rabbit anti-RPA (kindly donated by Dr. Edita Marcon) 1:100 dilution, and mouse anti-MLH1 (BD Bioscience) 1:50 dilution, goat anti-RNF212 (kindly donated by Dr. N. Hunter) 1:150 dilution, rabbit anti-p-S271-HORMAD2 (kindly donated by Dr. A. Toth) 1:250 dilution, rabbit anti-p-S33-RPA32 (Bethyl) 1:100 dillution, rabbit anti-pS317-CHK1 (Cell Signaling) 1:25 dillution. These antibodies have been routinely used to study meiotic progression in mammals ${ }^{50,58}$. Combined immunofluorescence/FISH was performed using a BAC probe RP24-204O18 (CHORI BACPAC library) labeled with digoxigenin to detect the X-linked Scml2 gene. After immunostaining, slides were treated with a pre-warmed denaturation solution ( $70 \%$ formamide in $2 \% \mathrm{SSC}, \mathrm{pH} 7.2-7.4$ ) for $20 \mathrm{~min}$ at $74^{\circ} \mathrm{C}$, incubated in a humid chamber for $2.5 \mathrm{~h}$ at $65^{\circ} \mathrm{C}$ with $100 \mu \mathrm{l}$ of $1 \mathrm{M}$ sodium thiocyanate, and immediately immersed in denaturation solution for $20 \mathrm{~min}$ at $74^{\circ} \mathrm{C}$. Slides were dehydrated in a series of ethanol solutions. A volume of $5 \mu \mathrm{l}$ of probe was mixed with one volume of hybridization buffer $(4 \times$ SSC, $50 \%$ dextran sulfate, $2 \mathrm{mg} / \mathrm{ml} \mathrm{BSA}, 2 \mathrm{mM}$ vanadyl ribonucleoside), denatured for $10 \mathrm{~min}$ at $75^{\circ} \mathrm{C}$ and reannealed for $10 \mathrm{~min}$ at $37^{\circ} \mathrm{C}$. Slides were incubated with hybridization mix for $72 \mathrm{~h}$ at $37^{\circ} \mathrm{C}$. Slide posthybridization treatments consisted of three washes for $5 \mathrm{~min}$ in washing solution (50\% formamide, $2 \times \mathrm{SSC} \mathrm{pH} 7.2-7.4$ ) at $45^{\circ} \mathrm{C}$, three washes for $5 \mathrm{~min}$ in $2 \times \mathrm{SSC}$ at $45^{\circ} \mathrm{C}$, one immersion in $4 \times$ SSC, $0.1 \%$ Tween 20 at room temperature, one incubation for $30 \mathrm{~min}$ with blocking buffer $(4 \times \mathrm{SCC}, 4 \mathrm{mg} / \mathrm{ml} \mathrm{BSA}, 0.1 \%$ Tween 20) at $37^{\circ} \mathrm{C}$, one incubation for $1 \mathrm{~h}$ with $30 \mu \mathrm{l}$ of digoxigenin detection solution (10\% Anti-Digoxigenin-Fluorescein (ApopTag Plus Fluorescein In Situ Apoptosis Detection kit (Millipore)) in $4 \times \mathrm{SSC}, 0.1 \%$ Tween 20 ) at $37^{\circ} \mathrm{C}$, and three washes for $5 \mathrm{~min}$ in $4 \times$ SSC, $0.1 \%$ Tween 20 at room temperature. DAPI (4, 6 -diamidino2-phenylindole) (Sigma) was used to stain the DNA.

RNA-FISH and immunofluorescence. RNA FISH was carried out using BAC probe bMQ-372M23 (Mouse bMQ BAC library) labeled with digoxigenin to detect the $Z f x$ gene, as previously described ${ }^{59}$. Briefly, BAC-containing bacteria were grown in an overnight LB-Chloramphenicol culture at $37^{\circ} \mathrm{C}$ and a standard miniprep method was used to isolate BAC DNA. $2 \mu \mathrm{g}$ of BAC DNA were labeled using DIG-Nick Translation Mix (Roche) and precipitated with Cot-1 DNA (Invitrogen) and salmon sperm DNA (Stratagene). Mouse testes were minced, then cells were treated with CSK buffer $(100 \mathrm{mM} \mathrm{NaCl}, 300 \mathrm{mM}$ sucrose, $3 \mathrm{mM} \mathrm{MgCl} 2$, $10 \mathrm{mM}$ PIPES, $0.5 \%$ Triton X-100, $2 \mathrm{mM}$ vanadyl ribonucleoside (New England Biolabs)) and fixed in 4\% paraformaldehyde in PBS, and slides were dehydrated in a series of ethanol solutions. $30 \mu \mathrm{l}$ of probe was denatured for $10 \mathrm{~min}$ at $80^{\circ} \mathrm{C}$ and reannealed for $30 \mathrm{~min}$ at $37^{\circ} \mathrm{C}$. Slides were incubated overnight at $37^{\circ} \mathrm{C}$. Slide posthybridization treatments consisted of three washes for $5 \mathrm{~min}$ in $50 \%$ formamide, $2 \times \operatorname{SSC} \mathrm{pH} 7.2-7.4$ solution at $45^{\circ} \mathrm{C}$, three washes for $5 \mathrm{~min}$ in $2 \times \mathrm{SSC}$ at $45^{\circ} \mathrm{C}$ one immersion in $4 \times$ SSC, $0.1 \%$ Tween 20 at room temperature, one incubation for 30 min with blocking buffer $(4 \times \mathrm{SCC}, 4 \mathrm{mg} / \mathrm{ml} \mathrm{BSA}, 0.1 \%$ Tween- 20$)$ at $37^{\circ} \mathrm{C}$, and one incubation for $1 \mathrm{~h}$ with anti-digoxigenin-FITC (1:10, Millipore). RNA FISH was then followed by immunostaining with an anti-HORMAD1 antibody (Abcam) at 1:50 dilution. Cells were examined on an Olympus IX70 inverted microscope. Images were captured using a computer-assisted (DeltaVision) CCD camera (Photometrics). Pachytene cells were defined based on continuous HORMAD1 staining along the X- and Y-chromosome axes.

SPO11-oligonucleotide complex detection and western blotting. Testis extract preparation, immunoprecipitation, SPO11-oligonucleotide detection and western blot analysis were performed essentially as previously described ${ }^{60}$. Briefly, SPO11oligonucleotide complexes and free SPO11 were isolated from testis lysates by two

Fig. 5 In vitro inhibition of ATR and CHK1 block recombination and prophase progression. a Percentage of SYCP3-positive cells in untreated testis fragments at 0,7 , and 14 days of culture. Columns and lines indicate the mean and SD from four replicates. $\mathbf{b}$ Mean percentage of spermatocytes at the indicated stages of prophase in untreated testis fragments from two replicates. c Proportion of SYCP3-positive cells at D14 in testis fragments treated with the indicated dosages of AZ20, PF-477736, or LY2603618, and their respective DMSO controls. Data obtained from three replicates per each condition. $P$ values are from Fisher's exact tests. d Proportion of spermatocytes at different stages of meiotic prophase at D14 of culture. Data obtained from two replicates per each condition. $P$ values are from $G$ tests. e Representative images of spermatocytes from testes treated with DMSO3 or $5 \mu M$ AZ20 showing progression of meiotic prophase, followed by staining for SYCP3 and $\gamma H 2 A X . \mathbf{f}$ RAD51 foci at the indicated stages from D14 cultures (Color code key is in panel $\mathbf{i}$ ). In this and other graphs of focus counts, horizontal lines denote the means and $p$ values are from pairwise $t$ tests. $\mathbf{g}$, $\mathbf{h}$ Representative spermatocytes from cultured samples stained for SYCP3 and either RAD51 (g) or DMC1 (h). i RPA foci in cultured spermatocytes. $\mathbf{j}$ RAD51 foci per spermatocyte from control and PF-477736- or LY2603618-treated samples. k Representative images of spermatocytes from D14 cultures treated with DMSO or $1 \mu$ M PF-477736 and immunostained for SYCP3 and RAD51. Data presented for each culture condition correspond to at least two experiments performed using different samples. Scale bars in all micrographs represent $10 \mu \mathrm{m}$ 
a

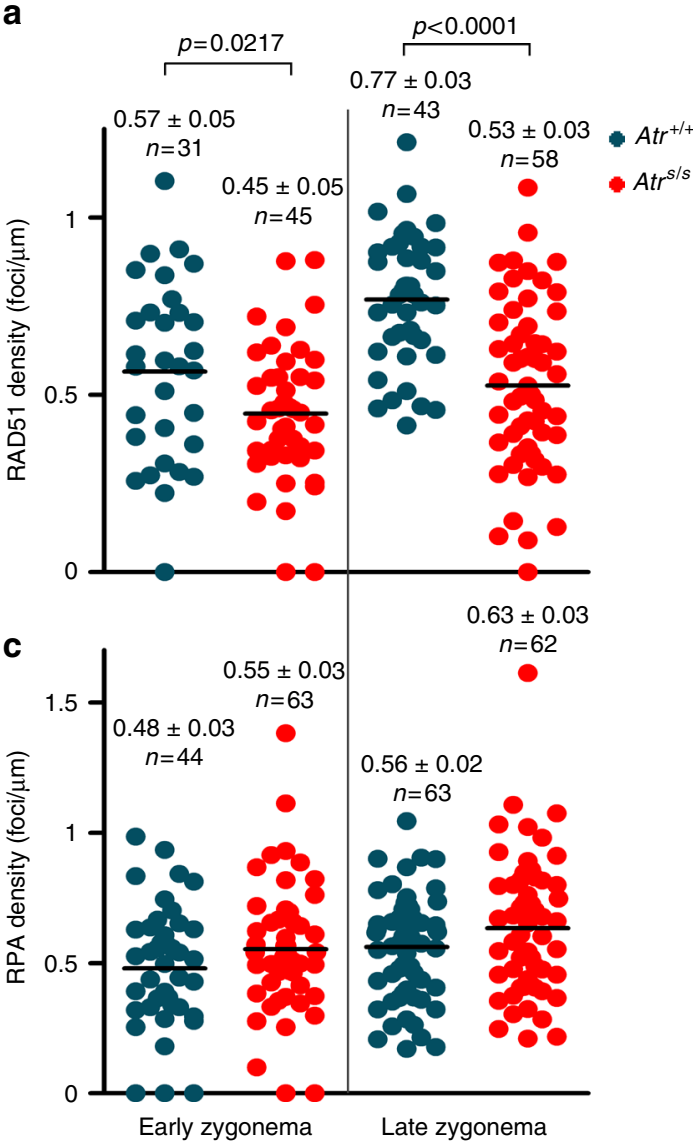

b

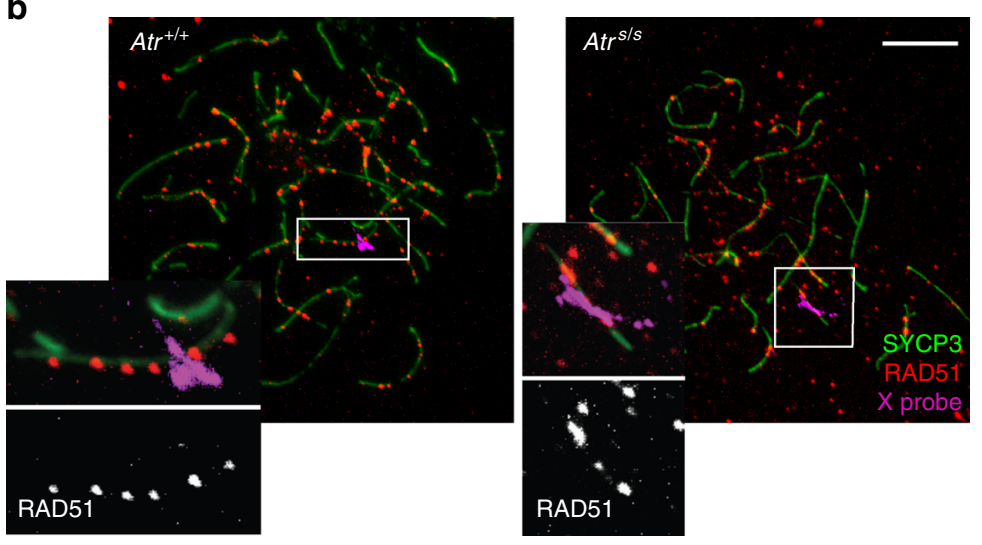

d
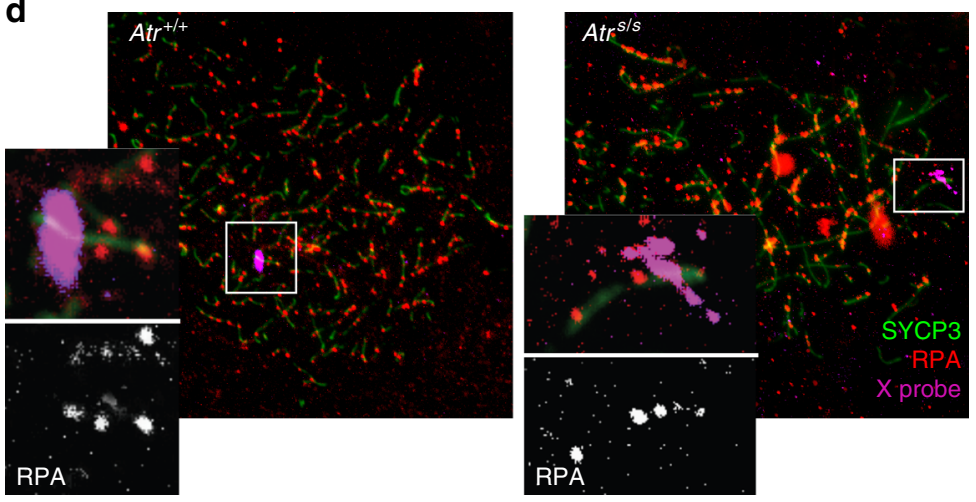

e

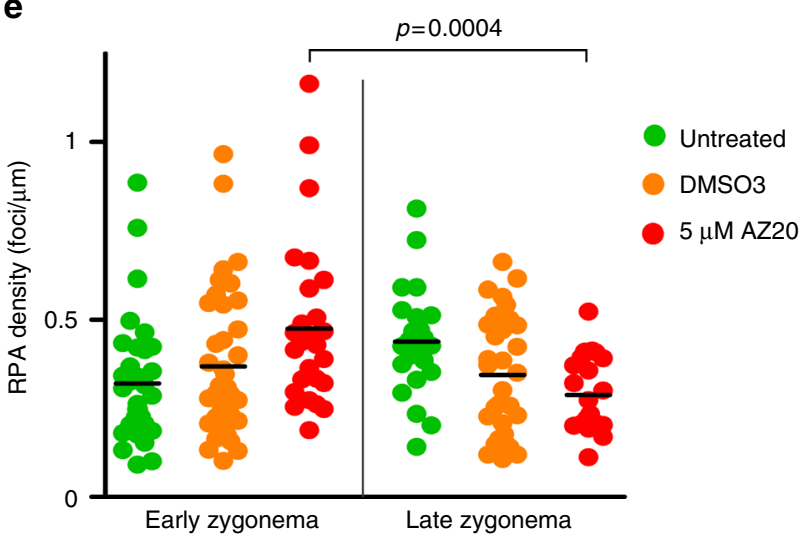

Fig. 6 ATR function is needed for normal levels of recombination markers on unsynapsed axes. a RAD51 focus density along the entire measurable Xchromosome axis. Horizontal black lines denote the means. $P$ values are from $t$ tests. $\mathbf{b}$ Representative images of late zygotene spermatocytes. Images show overlays of immunofluorescence against SYCP3 and RAD51 with fluorescence in situ hybridization (FISH) for the X-PAR boundary. Scale bar $=10 \mu \mathrm{m}$. Inset images show RAD51 foci on the measurable X-chromosome axis. c RPA focus density on the measurable $X$ chromosome. $\mathbf{d}$ Images of early zygotene spermatocytes showing immunofluorescence against SYCP3 and RPA, overlaid with FISH for X-PAR boundary. Inset images show RPA foci on the measurable X-chromosome axis. e RPA focus numbers present on unsynapsed axes at D14 from spermatocytes cultured in vitro

rounds of immunoprecipitation with an anti-SPO11 monoclonal antibody (Spol1180) on Protein A-agarose beads (Sigma). SPO11-oligonucleotide complexes were labeled with $\left[\alpha^{-32} \mathrm{P}\right]$ dCTP using terminal deoxynucleotidyl transferase (Fisher), released by boiling in Laemmli buffer and fractionated by SDS-PAGE. The electrophoresed products were transferred onto polyvinylidene fluoride (PVDF) membrane. Radiolabeled species were detected using Fuji phosphor screens and quantified with ImageGauge software. The membrane was probed with the SPO11 antibody at 1:2000 dilution.

For western blot analysis, wild type and Seckel mouse testis protein extracts were obtained by mincing the tissues in RIPA buffer and boiling them for $10 \mathrm{~min}$. After centrifugation, supernatants were run in a $4-20 \%$ gradient SDS-PAGE. Transfer to a nitrocellulose membrane was performed using a Trans-Blot Turbo System (Bio Rad).
The membrane was probed with ATR antibody (Abcam, 1:15000 dilution) and tubulin antibody (Abcam, 1:5000 dilution).

In vivo drug administration. AZ20 (Selleckchem) compound dissolved in $10 \%$ $\mathrm{DMSO} / 40 \%$ propylene glycol $/ 50 \%$ water was administered orally to adult wild-type males at a single daily dose of $50 \mathrm{mg} / \mathrm{kg}$ for 3 or 7 consecutive days or to Spo $11^{-/}$ males for 7 days. Control animals were administrated with the same volume of vehicle solution. Allocation of treated and control animals to each group was performed randomly. Animals were killed $24 \mathrm{~h}$ after the treatment ended.

Neonatal testis organ culture. Five dpp mouse testes were cultured as previously described $^{61}$. Briefly, $1.5 \%$ agarose gel cubes were placed in a six-well plate and 
pre-soaked overnight in culture medium (10\% KSR (Invitrogen), 1\% AntibioticAntimycotic $100 \times($ Gibco), $0.37 \%$ sodium bicarbonate in $\alpha$-MEM (Invitrogen) in a culture incubator supplied with $5 \% \mathrm{CO}_{2}$ in air and maintained at $34^{\circ} \mathrm{C}$. On the day of the culture, medium from the six-well plates was removed and replaced with fresh culture medium. Testis fragments from $5 \mathrm{dpp}$ mice were placed on top of the agarose cubes and incubated for 7 days with $5 \% \mathrm{CO}_{2}$ in air and $34{ }^{\circ} \mathrm{C}$. Samples were then treated with $0.2,1$, or $5 \mu \mathrm{M}$ AZ20, $1 \mu \mathrm{M}$ PF-477736 or $1 \mu \mathrm{M}$ LY2603618 (all drugs from Selleckchem) dissolved in DMSO (Sigma). Control samples were treated with equivalent volumes of DMSO used for each inhibitor concentration. Testis fragments were incubated with $5 \% \mathrm{CO}_{2}$ in air at $34{ }^{\circ} \mathrm{C}$ for 7 more days. Meiotic spreads from cultured testis fragments were performed at day 0,7 , or 14 of culture. Surface spreads were immunostained to analyze meiotic progression and recombination.

SC length, MLH1 distribution, and recombination focus density. Chromosomes axis length, SC length, and recombination focus position along the axis/SCs were recorded using MicroMeasure, as previously published ${ }^{20}$. Briefly, positions of MLH1 foci were recorded as the distance from each focus to the centromeric end of the chromosome, identified by the brighter DAPI staining of pericentromeric heterochromatin. MLH1 positions were expressed as a percentage of the total chromosome (or SC) length and are presented as cumulative graphs (Figs. $3 \mathrm{e}$ and 4i). Similarly, to measure cytological interference, the distances between adjacent MLH1 foci (IF, interfocal distances) from those SCs displaying more than one MLH1 focus were expressed as a percentage of the SC length and presented as cumulative graphs (Figs. $3 \mathrm{f}$ and $4 \mathrm{j}$ ). Recombination focus density was obtained by dividing the number of recombination foci by the chromosome axis length in micrometers.

Statistical analysis. Student's $t$ tests, Mann-Whitney tests and one-way ANOVA tests were performed using GraphPad Prism software and/or GraphPad QuickCalcs online resource (http://www.graphpad.com/quickcalcs/).

Image processing and data analysis. Microscopy analysis was performed blindly whenever possible. Nonetheless, mutant and treated samples displayed a particular phenotype (see Results section) that allowed their identification. Image acquisition was performed using a Zeiss Axioskop microscope connected with a ProgRes Jenoptik camera. Images were captured using ProgRes CapturePro software and were processed using Photoshop and ImageJ to quantify fluorescence intensity and chromosome axes length and/or MicroMeasure version 3.3 to analyze MLH1 focus position and axis length, as previously described ${ }^{20}$.

Data availability. All relevant data are presented in the figures or supplementary figures and tables and are available from the authors upon request.

Received: 22 May 2017 Accepted: 24 May 2018

Published online: 05 July 2018

\section{References}

1. Keeney, S., Giroux, C. N. \& Kleckner, N. Meiosis-specific DNA double-strand breaks are catalyzed by Spo11, a member of a widely conserved protein family. Cell 88, 375-384 (1997).

2. Baudat, F., Imai, Y. \& de Massy, B. Meiotic recombination in mammals: localization and regulation. Nat. Rev. Genet. 14, 794-806 (2013).

3. Cimprich, K. A. \& Cortez, D. ATR: an essential regulator of genome integrity. Nat. Rev. Mol. Cell Biol. 9, 616-627 (2008).

4. Maréchal, A. \& Zou, L. DNA damage sensing by the ATM and ATR kinases. Cold Spring Harb. Perspect. Biol. 5, a012716 (2013).

5. Royo, H. et al. ATR acts stage specifically to regulate multiple aspects of mammalian meiotic silencing. Genes Dev. 27, 1484-1494 (2013).

6. Burgoyne, P. S., Mahadevaiah, S. K. \& Turner, J. M. A. The consequences of asynapsis for mammalian meiosis. Nat. Rev. Genet. 10, 207-216 (2009).

7. Royo, H. et al. Evidence that meiotic sex chromosome inactivation is essential for male fertility. Curr. Biol. 20, 2117-2123 (2010).

8. Bellani, M. A., Romanienko, P. J., Cairatti, D. A. \& Camerini-Otero, R. D. SPO11 is required for sex-body formation, and Spo11 heterozygosity rescues the prophase arrest of Atm-/- spermatocytes. J. Cell Sci. 118, 3233-3245 (2005).

9. Barchi, M. et al. Surveillance of different recombination defects in mouse spermatocytes yields distinct responses despite elimination at an identical developmental stage. Mol. Cell Biol. 25, 7203-7215 (2005).

10. Subramanian, V. V. \& Hochwagen, A. The meiotic checkpoint network: stepby-step through meiotic prophase. Cold Spring Harb. Perspect. Biol. 6, a016675 (2014).

11. Kurzbauer, M.-T., Uanschou, C., Chen, D. \& Schlögelhofer, P. The recombinases DMC1 and RAD51 are functionally and spatially separated during meiosis in Arabidopsis. Plant Cell 24, 2058-2070 (2012).
12. Moens, P. B. et al. The association of ATR protein with mouse meiotic chromosome cores. Chromosoma 108, 95-102 (1999).

13. Brown, E. J. \& Baltimore, D. ATR disruption leads to chromosomal fragmentation and early embryonic lethality. Genes Dev. 14, 397-402 (2000).

14. Murga, M. et al. A mouse model of ATR-Seckel shows embryonic replicative stress and accelerated aging. Nat. Genet. 41, 891-898 (2009).

15. O'Driscoll, M. Mouse models for ATR deficiency. DNA Repair 8, 1333-1337 (2009).

16. O’Driscoll, M., Ruiz-Perez, V. L., Woods, C. G., Jeggo, P. A. \& Goodship, J. A. A splicing mutation affecting expression of ataxia-telangiectasia and Rad3related protein (ATR) results in Seckel syndrome. Nat. Genet. 33, 497-501 (2003).

17. Ichijima, Y. et al. MDC1 directs chromosome-wide silencing of the sex chromosomes in male germ cells. Genes Dev. 25, 959-971 (2011).

18. Fedoriw, A. M., Menon, D., Kim, Y., Mu, W. \& Magnuson, T. Key mediators of somatic ATR signaling localize to unpaired chromosomes in spermatocytes. Development 142, 2972-2980 (2015).

19. Mahadevaiah, S. K. et al. Recombinational DNA double-strand breaks in mice precede synapsis. Nat. Genet. 27, 271-276 (2001).

20. Barchi, M. et al. ATM promotes the obligate XY crossover and both crossover control and chromosome axis integrity on autosomes. PLOS Genet. 4, e1000076 (2008).

21. Kauppi, L. et al. Distinct properties of the XY pseudoautosomal region crucial for male meiosis. Science 331, 916-920 (2011).

22. Bannister, L. A. \& Schimenti, J. C. Homologous recombinational repair proteins in mouse meiosis. Cytogenet. Genome Res. 107, 191-200 (2004).

23. Brown, M. S., Grubb, J., Zhang, A., Rust, M. J. \& Bishop, D. K. Small Rad51 and Dmcl complexes often co-occupy both ends of a meiotic DNA double strand break. PLOS Genet. 11, e1005653 (2015).

24. Pan, J. \& Keeney, S. Detection of SPO11-oligonucleotide complexes from mouse testes. Methods Mol. Biol. 557, 197-207 (2009).

25. Pittman, D. L. et al. Meiotic prophase arrest with failure of chromosome synapsis in mice deficient for Dmc1, a germline-specific RecA homolog. Mol. Cell 1, 697-705 (1998).

26. De Vries, S. S. et al. Mouse MutS-like protein Msh5 is required for proper chromosome synapsis in male and female meiosis. Genes Dev. 13, 523-531 (1999).

27. Edelmann, W. et al. Meiotic pachytene arrest in MLH1-deficient mice. Cell 85, 1125-1134 (1996).

28. Moens, P. B., Marcon, E., Shore, J. S., Kochakpour, N. \& Spyropoulos, B. Initiation and resolution of interhomolog connections: crossover and noncrossover sites along mouse synaptonemal complexes. J. Cell Sci. 120, 1017-1027 (2007).

29. Bartrand, A. J., Iyasu, D., Marinco, S. M. \& Brush, G. S. Evidence of meiotic crossover control in Saccharomyces cerevisiae through Mec1-mediated phosphorylation of replication protein A. Genetics 172, 27-39 (2006).

30. Serrentino, M.-E., Chaplais, E., Sommermeyer, V. \& Borde, V. Differential association of the conserved SUMO ligase Zip3 with meiotic double-strand break sites reveals regional variations in the outcome of meiotic recombination. PLOS Genet. 9, e1003416 (2013).

31. Reynolds, A. et al. RNF212 is a dosage-sensitive regulator of crossing-over during mammalian meiosis. Nat. Genet. 45, 269-278 (2013).

32. Anderson, L. K., Reeves, A., Webb, L. M. \& Ashley, T. Distribution of crossing over on mouse synaptonemal complexes using immunofluorescent localization of MLH1 protein. Genetics 151, 1569-1579 (1999).

33. Berchowitz, L. E. \& Copenhaver, G. P. Genetic interference: don't stand so close to me. Curr. Genom. 11, 91-102 (2010).

34. De Boer, E., Stam, P., Dietrich, A. J. J., Pastink, A. \& Heyting, C. Two levels of interference in mouse meiotic recombination. Proc. Natl Acad. Sci. USA 103, 9607-9612 (2006)

35. Foote, K. M. et al. Discovery of 4-\{4-[(3R)-3-Methylmorpholin-4-yl]-6-[1(methylsulfonyl)cyclopropyl]pyrimidin-2-yl $\}-1 \mathrm{H}$-indole (AZ20): a potent and selective inhibitor of ATR protein kinase with monotherapy in vivo antitumor activity. J. Med. Chem. 56, 2125-2138 (2013).

36. Goetz, P., Chandley, A. C. \& Speed, R. M. Morphological and temporal sequence of meiotic prophase development at puberty in the male mouse. $J$. Cell Sci. 65, 249-263 (1984).

37. Pacheco, S. et al. The ATM signaling cascade promotes recombinationdependent pachytene arrest in mouse spermatocytes. PLOS Genet. 11, e1005017 (2015).

38. Mital, P., Hinton, B. T. \& Dufour, J. M. The blood-testis and blood-epididymis barriers are more than just their tight junctions. Biol. Reprod. 84, 851-858 (2011).

39. Toledo, L. I., Murga, M. \& Fernandez-Capetillo, O. Targeting ATR and Chk1 kinases for cancer treatment: a new model for new (and old) drugs. Mol. Oncol. 5, 368-373 (2011).

40. Sørensen, C. S. et al. The cell-cycle checkpoint kinase Chk1 is required for mammalian homologous recombination repair. Nat. Cell Biol. 7, 195-201 (2005). 
41. Blasina, A. et al. Breaching the DNA damage checkpoint via PF-00477736, a novel small-molecule inhibitor of checkpoint kinase 1. Mol. Cancer Ther. 7, 2394-2404 (2008).

42. Dai, Y. \& Grant, S. New insights into checkpoint kinase 1 in the DNA damage response signaling network. Clin. Cancer Res. 16, 376-383 (2010).

43. Baudat, F., Manova, K., Yuen, J. P., Jasin, M. \& Keeney, S. Chromosome synapsis defects and sexually dimorphic meiotic progression in mice lacking Spo11. Mol. Cell 6, 989-998 (2000).

44. Carballo, J. A., Johnson, A. L., Sedgwick, S. G. \& Cha, R. S. Phosphorylation of the axial element protein Hop1 by Mec1/Tel1 ensures meiotic interhomolog recombination. Cell 132, 758-770 (2008).

45. Grushcow, J. M. et al. Saccharomyces cerevisiae checkpoint genes MEC1, RAD17 and RAD24 are required for normal meiotic recombination partner choice. Genetics 153, 607-620 (1999).

46. Wojtasz, L. et al. Mouse HORMAD1 and HORMAD2, two conserved meiotic chromosomal proteins, are depleted from synapsed chromosome axes with the help of TRIP13 AAA-ATPase. PLOS Genet. 5, e1000702 (2009).

47. Daniel, K. et al. Meiotic homologue alignment and its quality surveillance are controlled by mouse HORMAD1. Nat. Cell Biol. 13, 599-610 (2011).

48. Kauppi, L. et al. Numerical constraints and feedback control of double-strand breaks in mouse meiosis. Genes Dev. 27, 873-886 (2013).

49. Thacker, D., Mohibullah, N., Zhu, X. \& Keeney, S. Homologue engagement controls meiotic DNA break number and distribution. Nature 510, 241-246 (2014).

50. Roig, I. et al. Mouse TRIP13/PCH2 is required for recombination and normal higher-order chromosome structure during meiosis. PLOS Genet. 6, e1001062 (2010).

51. Keeney, S., Lange, J. \& Mohibullah, N. Self-organization of meiotic recombination initiation: general principles and molecular pathways. Аnnu. Rev. Genet. 48, 187-214 (2014).

52. Widger, A. et al. ATR is a multifunctional regulator of male mouse meiosis. Nat. Commun. https://doi.org/10.1038/s41467-018-04850-0 (2018).

53. Brown, M. S. \& Bishop, D. K. DNA strand exchange and RecA homologs in meiosis. Cold Spring Harb. Perspect. Biol. 7, a016659 (2015).

54. Dai, J., Voloshin, O., Potapova, S. \& Camerini-Otero, R. D. Meiotic knockdown and complementation reveals essential role of RAD51 in mouse spermatogenesis. Cell Rep. 18, 1383-1394 (2017).

55. Fukuda, T. et al. Phosphorylation of chromosome core components may serve as axis marks for the status of chromosomal events during mammalian meiosis. PLOS Genet. 8, el002485 (2012).

56. Gray, S., Allison, R. M., Garcia, V., Goldman, A. S. H. \& Neale, M. J. Positive regulation of meiotic DNA double-strand break formation by activation of the DNA damage checkpoint kinase Mec1(ATR). Open Biol. 3, 130019 (2013).

57. Ahmed, E. A., Sfeir, A., Takai, H. \& Scherthan, H. Ku70 and non-homologous end joining protect testicular cells from DNA damage. J. Cell Sci. 126, 3095-3104 (2013).

58. Roig, I. et al. Female-specific features of recombinational double-stranded DNA repair in relation to synapsis and telomere dynamics in human oocytes. Chromosoma 113, 22-33 (2004).

59. Mahadevaiah, S. K., Costa, Y. \& Turner, J. M. A. Using RNA FISH to study gene expression during mammalian meiosis. Methods Mol. Biol. 558, 433-444 (2009).

60. Lange, J. et al. ATM controls meiotic double-strand-break formation. Nature 479, 237-240 (2011).

61. Sato, T. et al. In vitro production of functional sperm in cultured neonatal mouse testes. Nature 471, 504-507 (2011).

62. Inselman, A., Eaker, S. \& Handel, M. A. Temporal expression of cell cyclerelated proteins during spermatogenesis: establishing a timeline for onset of the meiotic divisions. Cytogenet. Genome Res. 103, 277-284 (2003).

63. Romanienko, P. J. \& Camerini-Otero, R. D. The mouse Spo11 gene is required for meiotic chromosome synapsis. Mol. Cell 6, 975-987 (2000).
64. Neale, M. J., Pan, J. \& Keeney, S. Endonucleolytic processing of covalent protein-linked DNA double-strand breaks. Nature 436, 1053-1057 (2005).

\section{Acknowledgements}

We thank M. A. Handel (The Jackson Laboratory, Bar Harbor, USA) for the anti-H1T antibody; E. Marcon for the anti-RPA antibody (University of Toronto, Canada); A. Toth for the anti-pHORMAD2 antibody (U. Dresden, Germany) and N. Hunter for the antiRNF212 antibody (UC Davis, USA); J. Turner (National Institute for Medical Research, London, UK) for assistance in the RNA-FISH experiments, for the X chromosome probe, for providing $A t r^{F L /}-$ testis samples and for sharing unpublished data; L. Kauppi (University of Helsinki, Finland) for providing us with protocols for the testis cultures; and members of the Roig lab and the Spanish Ministerio de Ciencia e Innovación-funded Network of Spanish groups working on Meiosis (MeioNet, BFU2015-71786-REDT) and Enrique Martínez Pérez (Imperial College, London, UK) for helpful discussions. M.M.O. was supported by a FPI fellowship from the Ministerio de Ciencia e Innovación (BES2011-045381). J.L. was supported in part by American Cancer Society post-doctoral fellowship (PF-12-157-01-DMC). S.K. is an Investigator of the Howard Hughes Medical Institute. This work was supported by the Ministerio de Ciencia e Innovación (BFU201018965, BFU2013-43965-P and BFU2016-80370-P, I.R.), by the UAB-Aposta award to young investigators (APOSTA2011-03, I.R.) and by the NIH (R35 GM118175, to M.J. and R35 GM118092 to S.K.).

\section{Author contributions}

S.P., M.M.-O., and I.R. conceived the experiments. O.F.-C. provided critical reagents. S. P., A.M.-L., M.M.-O., C.R., A.M.-M., J.F.-L., J.L., and I.R. performed the experiments. S, P., A.M.-L., M.M.-O., J.L., M.J., S.K., O.F.-C., M.G.-C., and I.R. analyzed the data. S.P., J. L., S.K., and I.R. wrote the manuscript.

\section{Additional information}

Supplementary Information accompanies this paper at https://doi.org/10.1038/s41467 018-04851-z.

Competing interests: The authors declare no competing interests.

Reprints and permission information is available online at http://npg.nature.com/ reprintsandpermissions/

Publisher's note: Springer Nature remains neutral with regard to jurisdictional claims in published maps and institutional affiliations.

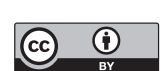

Open Access This article is licensed under a Creative Commons Attribution 4.0 International License, which permits use, sharing, adaptation, distribution and reproduction in any medium or format, as long as you give appropriate credit to the original author(s) and the source, provide a link to the Creative Commons license, and indicate if changes were made. The images or other third party material in this article are included in the article's Creative Commons license, unless indicated otherwise in a credit line to the material. If material is not included in the article's Creative Commons license and your intended use is not permitted by statutory regulation or exceeds the permitted use, you will need to obtain permission directly from the copyright holder. To view a copy of this license, visit http://creativecommons.org/ licenses/by/4.0/.

(c) The Author(s) 2018 